\title{
A Type of Multigrid Method Based on the Fixed-Shift Inverse Iteration for the Steklov Eigenvalue Problem
}

\author{
Feiyan Li and Hai Bi \\ School of Mathematical Sciences, Guizhou Normal University, Guiyang 550001, China \\ Correspondence should be addressed to Hai Bi; bihaimath@gznu.edu.cn
}

Received 17 March 2016; Revised 9 May 2016; Accepted 10 May 2016

Academic Editor: Luigi C. Berselli

Copyright (C) 2016 F. Li and H. Bi. This is an open access article distributed under the Creative Commons Attribution License, which permits unrestricted use, distribution, and reproduction in any medium, provided the original work is properly cited.

For the Steklov eigenvalue problem, we establish a type of multigrid discretizations based on the fixed-shift inverse iteration and study in depth its a priori/a posteriori error estimates. In addition, we also propose an adaptive algorithm on the basis of the a posteriori error estimates. Finally, we present some numerical examples to validate the efficiency of our method.

\section{Introduction}

Due to the wide applications in physical and mechanical field (see, e.g., [1-3]), there has been a lot of research on the numerical methods for Steklov eigenvalue problems; for instance, [4] studied the conforming linear finite element approximation, $[5,6]$ studied the nonconforming finite elements approximation, $[7,8]$ discussed a two-grid method of the conforming and nonconforming finite element method based on the inverse iteration, respectively, [9] studied multiscale asymptotic method, [10] studied multilevel method, [11] studied the spectral method, and [12] studied an adaptive algorithm based on the shifted inverse iteration.

In this paper we establish a type of multigrid discretizations based on the fixed-shift inverse iteration for the Steklov eigenvalue problem. The multilevel method in [10] made use of the inverse iteration and the extended finite element method. Compared with [10], our method has less computational complexity since we have no correction step in each iteration. On the other hand, compared with [12], we adopt the fixed-shift and thus avoid selecting appropriate shift to ensure the efficiency of shifted inverse iteration; meanwhile, we also do not face the difficulty of solving an almost singular algebraic system in the shifted inverse iteration.

We analyze elaborately the a priori and the a posteriori error estimates of the method proposed in this paper. Then, based on the a posteriori error estimates we design an adaptive algorithm of fixed-shift inverse iteration type.
Moreover, we also compare the performance of three types of multigrid methods. Numerical results illustrate that our method is also an efficient method for solving the Steklov eigenvalue problem.

The rest of this paper is organized as follows. In the subsequent section, some preliminaries needed in this paper are presented. In Section 3, a scheme of the inverse iteration with fixed-shift based on multigrid discretizations is established, and the a priori error estimates are also given. The a posteriori error estimates of the inverse iteration with fixed-shift are analyzed in Section 4. Numerical experiments are presented in the final section.

In this paper, $C$ with or without subscript denotes a constant independent of mesh size and iterative times.

\section{Preliminaries}

Consider the Steklov eigenvalue problem

$$
\begin{aligned}
-\Delta u+u & =0 \quad \text { in } \Omega, \\
\frac{\partial u}{\partial n} & =\lambda u \quad \text { on } \partial \Omega,
\end{aligned}
$$

where $\Omega \subset \mathbb{R}^{2}$ is a polygonal domain with $\theta$ being the largest inner angle of $\Omega$ and $\partial u / \partial n$ is the outward normal derivative.

We denote the real order Sobolev spaces with norm $\|\cdot\|_{t}$ and $\|\cdot\|_{t, \partial \Omega}$ by $H^{t}(\Omega)$ and $H^{t}(\partial \Omega)$, respectively; $H^{0}(\partial \Omega)=$ $L_{2}(\partial \Omega)$. 
The variational form of (1) is given by the following: find $\lambda \in \mathbb{R}$ and $u \in H^{1}(\Omega), u \neq 0$, such that

$$
a(u, v)=\lambda b(u, v), \quad \forall v \in H^{1}(\Omega),
$$

where

$$
\begin{aligned}
a(u, v) & =\int_{\Omega} \nabla u \cdot \nabla v+u v d x, \\
b(u, v) & =\int_{\partial \Omega} u v d s, \\
\|u\|_{b} & =b(u, u)^{1 / 2}=\|u\|_{0, \partial \Omega} .
\end{aligned}
$$

As we know, $a(\cdot, \cdot)$ is a symmetric, continuous, and $H^{1}(\Omega)$-elliptic bilinear form on $H^{1}(\Omega) \times H^{1}(\Omega)$. Thus, we use $a(\cdot, \cdot)$ and $\|\cdot\|_{a}=\sqrt{a(\cdot, \cdot)}=\|\cdot\|_{1}$ as the inner product and norm on $H^{1}(\Omega)$, respectively.

Let $H^{-1 / 2}(\partial \Omega)$ be the dual space of $H^{1 / 2}(\partial \Omega)$ with norm given by

$$
\|w\|_{-1 / 2, \partial \Omega}=\sup _{v \in H^{1 / 2}(\partial \Omega)} \frac{\langle w, v\rangle}{\|v\|_{1 / 2, \partial \Omega}},
$$

where $\langle w, v\rangle$ is the dual product on $H^{-1 / 2}(\partial \Omega) \times H^{1 / 2}(\partial \Omega)$. When $w \in L^{2}(\partial \Omega),\langle w, v\rangle=b(w, v)$.

Let $\left\{\pi_{h}\right\}$ be a family of regular triangulations of $\Omega$ with the mesh diameter $h$, and let $V_{h} \subset H^{1}(\Omega)$ be a space of piecewise polynomials defined on $\pi_{h}$. For any $w \in H^{1}(\Omega)$, the following conclusion holds:

$$
\lim _{h \rightarrow 0} \inf _{v \in V_{h}}\|w-v\|_{a}=0 .
$$

The conforming finite element approximation of (2) is the following: find $\lambda_{h} \in \mathbb{R}$ and $u_{h} \in V_{h}, u_{h} \neq 0$, such that

$$
a\left(u_{h}, v\right)=\lambda_{h} b\left(u_{h}, v\right), \quad \forall v \in V_{h} .
$$

Define the operators $T: H^{1}(\Omega) \rightarrow H^{1}(\Omega)$ and $T_{h}:$ $H^{1}(\Omega) \rightarrow V_{h} \subset H^{1}(\Omega)$ satisfying

$$
\begin{gathered}
a(T g, v)=b(g, v), \quad \forall v \in H^{1}(\Omega), \\
a\left(T_{h} g, v\right)=b(g, v), \quad \forall v \in V_{h} .
\end{gathered}
$$

Define the Ritz projection $P_{h}: H^{1}(\Omega) \rightarrow V_{h}$ by

$$
a\left(u-P_{h} u, v\right)=0, \quad \forall v \in V_{h} .
$$

From [13], we know that $\left\|T-T_{h}\right\|_{a} \rightarrow 0(h \rightarrow 0)$; (2) and (6) have the equivalent operator forms $T u=\mu u$ and $T_{h} u_{h}=$ $\mu_{h} u_{h}$, respectively, where $T_{h}=P_{h} T, \mu=1 / \lambda$, and $\mu_{h}=1 / \lambda_{h}$.

Suppose that $\lambda$ and $\lambda_{h}$ are the $k$ th eigenvalue of (2) and (6), respectively, and the algebraic multiplicity of $\lambda$ is equal to $q, \lambda=\lambda_{k}=\lambda_{k+1}=\cdots=\lambda_{k+q-1}$. Let $M(\lambda)$ be the space spanned by all eigenfunctions corresponding to $\lambda$ and let $M_{h}(\lambda)$ be the direct sum of eigenspaces corresponding to all eigenvalues of (6) that converge to $\lambda$. Let $\widehat{M}(\lambda)=\{v: v \in$ $\left.M(\lambda),\|v\|_{a}=1\right\}$.
Denote

$$
\begin{gathered}
\sigma(h)=\sup _{f \in H^{1}(\Omega),\|f\|_{a}=1} \inf _{v \in V_{h}}\|T f-v\|_{a}, \\
\rho(h)=\sup _{f \in L_{2}(\partial \Omega),\|f\|_{0, \partial \Omega}=1} \inf _{v \in V_{h}}\|T f-v\|_{a}, \\
\delta_{h}(\lambda)=\sup _{u \in \widehat{M}(\lambda)} \inf _{v \in V_{h}}\|u-v\|_{a} .
\end{gathered}
$$

It is obvious that $\delta_{h}(\lambda) \leq \lambda \sigma(h) \leq C \rho(h)$. It follows from Lemma 3.3 in [14] that

$$
\sigma(h) \longrightarrow 0 \quad(h \longrightarrow 0) .
$$

By using the trace theorem we have

$$
\|v\|_{0, \partial \Omega} \leq\|v\|_{1 / 2, \partial \Omega} \leq C_{1}\|v\|_{a}, \quad \forall v \in H^{1}(\Omega) .
$$

Moreover, if $v \in H^{1}(\Omega)$ and $w \in H^{1 / 2}(\partial \Omega)$ we know that $\langle v, w\rangle=b(v, w) \leq\|v\|_{0, \partial \Omega}\|w\|_{0, \partial \Omega}$ and, consequently,

$$
\|v\|_{-1 / 2, \partial \Omega} \leq\|v\|_{0, \partial \Omega} \leq C_{1}\|v\|_{a}, \quad \forall v \in H^{1}(\Omega) .
$$

For any $g \in H^{1}(\Omega), T_{h} g \in H^{1}(\Omega)$. Taking $v=T_{h} g$ in (8) we deduce

$$
\begin{aligned}
a\left(T_{h} g, T_{h} g\right) & =b\left(g, T_{h} g\right) \leq\|g\|_{-1 / 2, \partial \Omega}\left\|T_{h} g\right\|_{1 / 2, \partial \Omega} \\
& \leq C_{1}\|g\|_{-1 / 2, \partial \Omega}\left\|T_{h} g\right\|_{a},
\end{aligned}
$$

and thus we get

$$
\left\|T_{h} g\right\|_{a} \leq C_{1}\|g\|_{-1 / 2, \partial \Omega} .
$$

The following lemmas are needed in our analysis.

Lemma 1. Let $(\lambda, u)$ be an eigenpair of (2); then for any $v \in H^{1}(\Omega)$ with $\|v\|_{a}=1$, the Rayleigh quotient $R(v)=$ $a(v, v) /\|v\|_{b}^{2}$ satisfies

$$
R(v)-\lambda=\frac{\|v-u\|_{a}^{2}}{\|v\|_{b}^{2}}-\lambda \frac{\|v-u\|_{b}^{2}}{\|v\|_{b}^{2}} .
$$

Proof. See page 699 of [13].

Lemma 2. For any nonzero $u, v \in H^{1}(\Omega)$,

$$
\begin{aligned}
& \left\|\frac{u}{\|u\|_{a}}-\frac{v}{\|v\|_{a}}\right\|_{a} \leq 2 \frac{\|u-v\|_{a}}{\|u\|_{a}}, \\
& \left\|\frac{u}{\|u\|_{a}}-\frac{v}{\|v\|_{a}}\right\|_{a} \leq 2 \frac{\|u-v\|_{a}}{\|v\|_{a}} .
\end{aligned}
$$

Proof. See [15].

Lemma 3. Let $\lambda$ and $\lambda_{h}$ be the kth eigenvalue of (2) and (6), respectively. Then for any eigenfunction $u_{h}$ corresponding to $\lambda_{h}$ with $\left\|u_{h}\right\|_{a}=1$, there exist $u \in M(\lambda)$ and $h_{0}>0$ such that if $h \leq h_{0}$,

$$
\begin{aligned}
\left\|u_{h}-u\right\|_{a} & \leq C_{2} \delta_{h}(\lambda), \\
\left\|u_{h}-u\right\|_{0, \partial \Omega} & \leq C_{2} \rho(h) \delta_{h}(\lambda), \\
\left\|u_{h}-u\right\|_{-1 / 2, \partial \Omega} & \leq C_{2} \sigma(h) \delta_{h}(\lambda) ;
\end{aligned}
$$


for any $u \in \widehat{M}(\lambda)$, there exists $u_{h} \in M_{h}(\lambda)$ such that if $h \leq h_{0}$,

$$
\begin{gathered}
\left\|u-u_{h}\right\|_{a} \leq C_{3} \delta_{h}(\lambda), \\
\left\|u-u_{h}\right\|_{-1 / 2, \partial \Omega} \leq C_{3} \sigma(h) \delta_{h}(\lambda),
\end{gathered}
$$

where constants $C_{2}$ and $C_{3}$ are positive and only depend on $\lambda$.

Proof. See page 699 of [13] and Lemma 3.7 and (3.29b) of [14].

If $u \in M(\lambda), v \in H^{1}(\Omega),\|v\|_{a}=1$, and $\|v-u\|_{a} \leq$ $\left(4 \sqrt{\lambda} C_{1}\right)^{-1}$, then by Lemma 2 we have

$$
\begin{gathered}
\left\|v-\frac{u}{\|u\|_{a}}\right\|_{a} \leq 2\|v-u\|_{a} \leq\left(2 \sqrt{\lambda} C_{1}\right)^{-1}, \\
\left\|v-\frac{u}{\|u\|_{a}}\right\|_{0, \partial \Omega} \leq C_{1}\left\|v-\frac{u}{\|u\|_{a}}\right\|_{a} \leq \frac{1}{2 \sqrt{\lambda}} .
\end{gathered}
$$

From (2) we have $\|u /\| u\left\|_{a}\right\|_{0, \partial \Omega}=1 / \sqrt{\lambda}$; then

$$
\|v\|_{0, \partial \Omega} \geq\left\|\frac{u}{\|u\|_{a}}\right\|_{0, \partial \Omega}-\left\|v-\frac{u}{\|u\|_{a}}\right\|_{0, \partial \Omega} \geq \frac{1}{2 \sqrt{\lambda}} .
$$

Hence, from Lemma 1 we get

$$
|R(v)-\lambda| \leq 4 \lambda\left(1+\lambda C_{1}^{2}\right)\|v-u\|_{a}^{2} .
$$

Denote

$$
C_{4}=4 \lambda\left(1+\lambda C_{1}^{2}\right)
$$

and then when $\|v\|_{a}=1$ and $\|v-u\|_{a} \leq\left(4 \sqrt{\lambda} C_{1}\right)^{-1},(24)$ becomes

$$
|R(v)-\lambda| \leq C_{4}\|v-u\|_{a}^{2} .
$$

Since (6) implies $\lambda_{h}=R\left(u_{h}\right)$, then combining (26) and (18) we deduce that

$$
0 \leq \lambda_{h}-\lambda \leq C_{4}\left\|u_{h}-u\right\|_{a}^{2} \leq C_{4} C_{2}^{2} \delta_{h}^{2}(\lambda)
$$

\section{A Priori Error Estimates of the Inverse Iteration with Fixed-Shift}

Let $\left\{V_{h_{i}}\right\}_{0}^{\infty}$ be a family of conforming finite element spaces that satisfy $V_{h_{0}}=V_{H}, V_{h_{i}} \subset V_{h_{i+1}} \subset H^{1}(\Omega)(i=0,1, \ldots)$, and $\sigma\left(h_{i}\right) \rightarrow 0(i \rightarrow \infty)$. Referring to [16], we establish the following scheme of the inverse iteration with fixed-shift based on multigrid discretizations.

Scheme 4 (the inverse iteration with fixed-shift based on multigrid discretizations). Given the iterative times $l$ and $i 0$. Execute the following.

Step 1. Solve (2) on $V_{H}$ : find $\left(\lambda_{H}, u_{H}\right) \in \mathbb{R} \times V_{H}$ such that $\left\|u_{H}\right\|_{a}=1$ and

$$
a\left(u_{H}, v\right)=\lambda_{H} b\left(u_{H}, v\right), \quad \forall v \in V_{H} .
$$

Step 2. Let $u^{h_{0}} \Leftarrow u_{H}, \lambda^{h_{0}} \Leftarrow \lambda_{H}, i \Leftarrow 1$.

Step 3. Solve a linear system on $V_{h_{i}}$ : find $u^{\prime} \in V_{h_{i}}$ such that

$$
a\left(u^{\prime}, v\right)-\lambda^{h_{i-1}} b\left(u^{\prime}, v\right)=b\left(u^{h_{i-1}}, v\right), \quad \forall v \in V_{h_{i}} ;
$$

set $u^{h_{i}}=u^{\prime} /\left\|u^{\prime}\right\|_{a}$.

Step 4. Compute the Rayleigh quotient

$$
\lambda^{h_{i}}=\frac{a\left(u^{h_{i}}, u^{h_{i}}\right)}{b\left(u^{h_{i}}, u^{h_{i}}\right)} .
$$

Step 5. If $i>i 0$, then $\lambda^{h_{i 0}} \Leftarrow \lambda^{h_{i-1}}, i \Leftarrow i+1$; turn to Step 6; else, $i \Leftarrow i+1$, and return to Step 3 .

Step 6. Solve a linear system on $V_{h_{i}}$ : find $u^{\prime} \in V_{h_{i}}$ such that

$$
a\left(u^{\prime}, v\right)-\lambda^{h_{i 0}} b\left(u^{\prime}, v\right)=b\left(u^{h_{i-1}}, v\right), \quad \forall v \in V_{h_{i}}
$$

$\operatorname{set} u^{h_{i}}=u^{\prime} /\left\|u^{\prime}\right\|_{a}$.

Step 7. Compute the Rayleigh quotient

$$
\lambda^{h_{i}}=\frac{a\left(u^{h_{i}}, u^{h_{i}}\right)}{b\left(u^{h_{i}}, u^{h_{i}}\right)} .
$$

Step 8. If $i=l$, then output $\left(\lambda^{h_{l}}, u^{h_{l}}\right)$ and stop; else, $i \Leftarrow i+1$, and return to Step 6.

Let $\left(\lambda_{H}, u_{H}\right)$ be the $k$ th eigenpair of (28); then $\left(\lambda^{h_{l}}, u^{h_{l}}\right)$ derived from Scheme 4 is the $k$ th eigenpair approximation of (2).

In the following analysis, we also denote $\left(\lambda_{H}, u_{H}\right)=$ $\left(\lambda_{k, H}, u_{k, H}\right)$ and $\left(\lambda^{h_{l}}, u^{h_{l}}\right)=\left(\lambda_{k}^{h_{l}}, u_{k}^{h_{l}}\right)$.

Now, we will analyze the a priori error estimates of Scheme 4.

Denote $\operatorname{dist}(u, S)=\inf _{v \in S}\|u-v\|_{a}$.

Our analysis makes use of the following lemma (see Lemma 4.1 in [16]) for the shifted inverse iteration method. Let $\left(\lambda_{k}, u_{k}\right)$ and $\left(\lambda_{k, h}, u_{k, h}\right)$ denote the $k$ th eigenpair of (2) and (6), respectively, and $\mu_{k}=1 / \lambda_{k}, \mu_{k, h}=1 / \lambda_{k, h}, M\left(\mu_{k}\right)=$ $M\left(\lambda_{k}\right)$, and $M_{h}\left(\mu_{k}\right)=M_{h}\left(\lambda_{k}\right)$.

Lemma 5. Let $\left(\mu_{0}, u_{0}\right)$ be an approximation for $\left(\mu_{k}, u_{k}\right)$, where $\mu_{0}$ is not an eigenvalue of $T_{h}$, and $u_{0} \in V_{h}$ with $\left\|u_{0}\right\|_{a}=1$. Suppose that

(C1) dist $\left(u_{0}, M_{h}\left(\mu_{k}\right)\right) \leq 1 / 2$;

(C2) $\left|\mu_{0}-\mu_{k}\right| \leq \rho / 4$ and $\left|\mu_{j, h}-\mu_{j}\right| \leq \rho / 4$, for $j=k-1, k, k+$ $q(j \neq 0)$, where $\rho=\min _{\mu_{j} \neq \mu_{k}}\left|\mu_{j}-\mu_{k}\right|$ is the separation constant of the eigenvalue $\mu_{k}$;

(C3) $u^{\prime} \in V_{h}, u_{k}^{h} \in V_{h}$ satisfy

$$
\begin{aligned}
\left(\mu_{0}-T_{h}\right) u^{\prime} & =u_{0}, \\
u_{k}^{h} & =\frac{u^{\prime}}{\left\|u^{\prime}\right\|_{a}} .
\end{aligned}
$$


Then

$$
\begin{aligned}
& \operatorname{dist}\left(u_{k}^{h}, M_{h}\left(\mu_{k}\right)\right) \\
& \quad \leq \frac{4}{\rho} \max _{k \leq j \leq k+q-1}\left|\mu_{0}-\mu_{j, h}\right| \operatorname{dist}\left(u_{0}, M_{h}\left(\mu_{k}\right)\right) .
\end{aligned}
$$

Let $\delta_{0}$ be a positive constant satisfying the following inequalities:

$$
\begin{aligned}
& \max \left\{1, C_{2}, C_{3}\right\} \delta_{0} \leq \min \left\{\frac{1}{2},\left(4 \sqrt{\lambda_{j}} C_{1}\right)^{-1}\right\}, \\
& 4 C_{1}\left(C_{1} C_{4} \delta_{0}^{2}+\lambda_{k} C_{1} \delta_{0}+\lambda_{k} C_{3} \delta_{0}^{2}\right. \\
& \left.\quad+C_{1} C_{5} q^{1 / 2} C_{4} C_{2}^{2} \delta_{0}^{2}\right) \leq \frac{1}{2}, \\
& \frac{\delta_{0}}{\left(\lambda_{k}-\delta_{0}\right) \lambda_{k}} \leq \frac{\rho}{4}, \quad \delta_{0} \leq \frac{\lambda_{k}}{2}, \\
& \frac{C_{4} C_{2}^{2}}{\lambda_{j}^{2}} \delta_{0}^{2} \leq \frac{\rho}{4}, \quad j=k-1, k, \ldots, k+q, \quad j \neq 0 .
\end{aligned}
$$

Condition 6. There exists $\bar{u} \in \widehat{M}\left(\lambda_{k}\right)$ for $j=k-1, k, k+q(j \neq$ 0) such that

$$
\begin{aligned}
\left\|u_{k}^{h_{l-1}}-\bar{u}\right\|_{a} & \leq \delta_{0}, \\
\left|\lambda_{0}-\lambda_{k}\right| & \leq \delta_{0}, \\
\delta_{h_{l}}\left(\lambda_{j}\right) & \leq \delta_{0}, \\
\sigma\left(h_{l}\right) & \leq \delta_{0},
\end{aligned}
$$

where $\lambda_{0}$ is an approximate eigenvalue of $\lambda_{k}, u_{k}^{h_{l-1}}$ is an approximate eigenfunction obtained by Scheme 4 , and $\rho$ is the separation constant of the eigenvalue $\mu_{k}=1 / \lambda_{k}$.

Let the eigenvectors $\left\{u_{j, h_{l}}\right\}_{k}^{k+q-1}$ be an orthonormal basis of $M_{h_{l}}\left(\lambda_{k}\right)$ with respect to $a(\cdot, \cdot)$, and denote

$$
u^{*}=\sum_{j=k}^{k+q-1} a\left(u_{k}^{h_{l}}, u_{j, h_{l}}\right) u_{j, h_{l}} .
$$

From Lemma 3, we know that there exist eigenvectors $\left\{u_{j}^{0}\right\}_{k}^{k+q-1} \subset M\left(\lambda_{k}\right)$ making $u_{j, h_{l}}-u_{j}^{0}$ satisfy (18), (19), and (20). Let

$$
u_{k}=\sum_{j=k}^{k+q-1} a\left(u_{k}^{h_{l}}, u_{j, h_{l}}\right) u_{j}^{0}
$$

and then $u_{k} \in M\left(\lambda_{k}\right)$ and

$$
u_{k}-u^{*}=\sum_{j=k}^{k+q-1} a\left(u_{k}^{h_{l}}, u_{j, h_{l}}\right)\left(u_{j}^{0}-u_{j, h_{l}}\right) .
$$

To estimate the error, we split

$$
u_{k}^{h_{l}}-u_{k}=\left(u_{k}^{h_{l}}-u^{*}\right)-\left(u_{k}-u^{*}\right) .
$$

Now, we will analyze the first term $u_{k}^{h_{l}}-u^{*}$.
Theorem 7. Let $\left(\lambda_{k}^{h_{l}}, u_{k}^{h_{l}}\right)$ be an approximate eigenpair obtained by Scheme 4 with $\lambda_{0}=\lambda_{k}^{h_{i 0}}$. Assume that Lemma 3 and Condition 6 hold; then

$$
\begin{aligned}
& \left\|u_{k}^{h_{l}}-u^{*}\right\|_{a} \leq \frac{C_{0}}{4}\left|\lambda_{0}-\lambda_{k}\right|\left\{\left|\lambda_{k}^{h_{l-1}}-\lambda_{k}\right|\right. \\
& \left.\quad+\left\|u_{k}^{h_{l-1}}-\bar{u}\right\|_{-1 / 2, \partial \Omega}+\sigma\left(h_{l}\right) \delta_{h_{l}}\left(\lambda_{k}\right)\right\}, \quad l \geq 1,
\end{aligned}
$$

where $C_{0}$ is independent of mesh parameters and $l$.

Proof. We use Lemma 5 to complete the proof. First, we will verify that the conditions of Lemma 5 are satisfied.

From Lemma 3, we know that, for any given $\bar{u} \in \widehat{M}\left(\lambda_{k}\right)$, there exists $\widetilde{u}_{k, h_{l}} \in M_{h_{l}}\left(\lambda_{k}\right)$ such that

$$
\begin{gathered}
\left\|\bar{u}-\tilde{u}_{k, h_{l}}\right\|_{a} \leq C_{3} \delta_{h_{l}}\left(\lambda_{k}\right), \\
\left\|\bar{u}-\tilde{u}_{k, h_{l}}\right\|_{-1 / 2, \partial \Omega} \leq C_{3} \sigma\left(h_{l}\right) \delta_{h_{l}}\left(\lambda_{k}\right),
\end{gathered}
$$

where

$$
\tilde{u}_{k, h_{l}}=\sum_{i=k}^{k+q-1} \alpha_{i} u_{i, h_{l}}
$$

$$
\left\|\tilde{u}_{k, h_{l}}\right\|_{a} \leq C_{5} .
$$

Select $\mu_{0}=1 / \lambda_{0}$ and $u_{0}=\lambda_{k}^{h_{l-1}} T_{h_{l}} u_{k}^{h_{l-1}} /\left\|\lambda_{k}^{h_{l-1}} T_{h_{l}} u_{k}^{h_{l-1}}\right\|_{a}$. Then, by (15) and (13) we have

$$
\begin{aligned}
& \left\|\lambda_{k}^{h_{l-1}} T_{h_{l}} u_{k}^{h_{l-1}}-\widetilde{u}_{k, h_{l}}\right\|_{a}=\| \lambda_{k}^{h_{l-1}} T_{h_{l}} u_{k}^{h_{l-1}} \\
& -\sum_{i=k}^{k+q-1} \lambda_{i, h_{l}} T_{h_{l}} \alpha_{i} u_{i, h_{l}}\left\|_{a} \leq C_{1}\right\| \lambda_{k}^{h_{l-1}} u_{k}^{h_{l-1}} \\
& -\sum_{i=k}^{k+q-1} \lambda_{i, h_{l}} \alpha_{i} u_{i, h_{l}}\left\|_{-1 / 2, \partial \Omega}=C_{1}\right\| \lambda_{k}^{h_{l-1}} u_{k}^{h_{l-1}}-\lambda_{k} u_{k}^{h_{l-1}} \\
& +\lambda_{k} u_{k}^{h_{l-1}}-\lambda_{k} \bar{u}+\lambda_{k} \bar{u}-\lambda_{k} \tilde{u}_{k, h_{l}}+\lambda_{k} \tilde{u}_{k, h_{l}} \\
& -\sum_{i=k}^{k+q-1} \lambda_{i, h_{l}} \alpha_{i} u_{i, h_{l}} \|_{-1 / 2, \partial \Omega} \leq C_{1}\left(C_{1}\left|\lambda_{k}^{h_{l-1}}-\lambda_{k}\right|\right. \\
& +\lambda_{k}\left\|u_{k}^{h_{l-1}}-\bar{u}\right\|_{-1 / 2, \partial \Omega}+\lambda_{k}\left\|\bar{u}-\widetilde{u}_{k, h_{l}}\right\|_{-1 / 2, \partial \Omega} \\
& \left.+\left\|\sum_{i=k}^{k+q-1}\left(\lambda_{k}-\lambda_{i, h_{l}}\right) \alpha_{i} u_{i, h_{l}}\right\|_{-1 / 2, \partial \Omega}\right),
\end{aligned}
$$

noting that $\left\|\tilde{u}_{k, h_{l}}\right\|_{a} \geq\|\bar{u}\|_{a}-\left\|\bar{u}-\tilde{u}_{k, h_{l}}\right\|_{a} \geq 1-C_{3} \delta_{h_{l}}\left(\lambda_{k}\right) \geq 1-$ $C_{3} \delta_{0} \geq 1 / 2$; then using Lemma 2, (46), the Cauchy-Schwartz inequality, (26), Condition 6, and (36) we obtain

$$
\begin{gathered}
\operatorname{dist}\left(u_{0}, M_{h_{l}}\left(\lambda_{k}\right)\right) \leq \| u_{0}-\frac{\tilde{u}_{k, h_{l}}}{\left\|\tilde{u}_{k, h_{l}}\right\|_{a} \|_{a}} \\
\quad \leq \frac{2}{\left\|\tilde{u}_{k, h_{l}}\right\|_{a}}\left\|\lambda_{k}^{h_{l-1}} T_{h_{l}} u_{k}^{h_{l-1}}-\tilde{u}_{k, h_{l}}\right\|_{a}
\end{gathered}
$$




$$
\begin{aligned}
& \leq 4 C_{1}\left(C_{1}\left|\lambda_{k}^{h_{l-1}}-\lambda_{k}\right|+\lambda_{k}\left\|u_{k}^{h_{l-1}}-\bar{u}\right\|_{-1 / 2, \partial \Omega}\right. \\
& \left.+\lambda_{k} C_{3} \sigma\left(h_{l}\right) \delta_{h_{l}}\left(\lambda_{k}\right)+C_{1} C_{5} q^{1 / 2} C_{4} C_{2}^{2} \delta_{h_{l}}^{2}\left(\lambda_{k}\right)\right) \\
& \leq 4 C_{1}\left(C_{1} C_{4} \delta_{0}^{2}+\lambda_{k} C_{1} \delta_{0}+\lambda_{k} C_{3} \delta_{0}^{2}\right. \\
& \left.+C_{1} C_{5} q^{1 / 2} C_{4} C_{2}^{2} \delta_{0}^{2}\right) \leq \frac{1}{2},
\end{aligned}
$$

and then Condition (C1) in Lemma 5 holds.

By using the same arguments in [16], it is clear that the other two conditions in Lemma 5 are valid.

Hence, we see that the conditions of Lemma 5 hold.

Then, by the same proof method in [16], we derive that

$$
\begin{aligned}
& \left\|u_{k}^{h_{l}}-u^{*}\right\|_{a} \leq \frac{16}{\rho} C_{1}\left(\frac{2}{\lambda_{k}^{2}}\left|\lambda_{0}-\lambda_{k}\right|\right. \\
& \left.\quad+\frac{2}{\lambda_{k}^{2}} C_{4} C_{2}^{2} \delta_{h_{l}}^{2}\left(\lambda_{k}\right)\right)\left(C_{1}\left|\lambda_{k}^{h_{l-1}}-\lambda_{k}\right|\right. \\
& \quad+\lambda_{k}\left\|u_{k}^{h_{l-1}}-\bar{u}\right\|_{-1 / 2, \partial \Omega}+\lambda_{k} C_{3} \sigma\left(h_{l}\right) \delta_{h_{l}}\left(\lambda_{k}\right) \\
& \left.+C_{1} C_{5} q^{1 / 2} C_{4} C_{2}^{2} \delta_{h_{l}}^{2}\left(\lambda_{k}\right)\right) .
\end{aligned}
$$

Noting that the constants $C_{1}, C_{2}, C_{3}, C_{4}, C_{5}$, and $\rho$ are independent of mesh parameters and $l$ and Condition 6 holds, then based on the above inequality we conclude that there exists a positive constant $C_{0}$ that is independent of mesh parameters and $l$ such that (44) holds. And we can have $\min \left\{C_{0} / 4, C_{0} C_{1} / 4\right\}>q^{1 / 2} C_{2}$. The proof is completed.

Next, we will analyze the error $u_{k}-u^{*}$.

Theorem 8. The error $u_{k}-u^{*}$ satisfies

$$
\begin{aligned}
\left\|u_{k}-u^{*}\right\|_{a} & \leq \frac{C_{0}}{4} \delta_{h_{l}}\left(\lambda_{k}\right), \\
\left\|u_{k}-u^{*}\right\|_{-1 / 2, \partial \Omega} & \leq \frac{C_{0} C_{1}}{4} \sigma\left(h_{l}\right) \delta_{h_{l}}\left(\lambda_{k}\right) .
\end{aligned}
$$

Proof. The estimates (51) and (52) can be obtained by the proof arguments in [16].

Based on the above two theorems, we now analyze the a priori error estimates of Scheme 4 .

Condition 9. For any given $\beta_{0}, \beta_{0}^{\prime} \in(0,1)$, there exist $0<$ $\beta_{0} \leq \beta_{i}<1$ and $0<\beta_{0}^{\prime} \leq \beta_{i}^{\prime}<1(i=1,2, \ldots)$ such that $\delta_{h_{i}}\left(\lambda_{k}\right)=\beta_{i} \delta_{h_{i-1}}\left(\lambda_{k}\right)$ and $\sigma\left(h_{i}\right)=\beta_{i}^{\prime} \sigma\left(h_{i-1}\right)$, respectively, $\sigma\left(h_{i}\right) \stackrel{\rightarrow}{\rightarrow}(i \rightarrow \infty)$.

In the practice Condition 9 is not a restrictive condition. For example, let $\pi_{h_{i}}$ be obtained from $\pi_{h_{i-1}}$ via regular refinement (producing 4 congruent elements) such that $h_{i}=$ $(1 / 2) h_{i-1}$; then, when $M\left(\lambda_{k}\right) \subset H^{1+\gamma}(\Omega)$ and $\{T f: f \in$ $\left.H^{1}(\Omega)\right\} \subset H^{1+\gamma}(\Omega)$ we have $\delta_{h_{i}}\left(\lambda_{k}\right) \approx(1 / 2)^{\gamma} \delta_{h_{i-1}}\left(\lambda_{k}\right)$ and $\sigma\left(h_{i}\right) \approx(1 / 2)^{\gamma} \sigma\left(h_{i-1}\right)$ (see [10]), where $\gamma=1$ if $\Omega$ is convex and $0<\gamma<1$ if $\Omega$ is concave.
Theorem 10. Let $\left(\lambda_{k}^{h_{l}}, u_{k}^{h_{l}}\right)$ be an approximate eigenpair obtained by Scheme 4. Suppose that Condition 9 holds; then there exist $u_{k} \in M\left(\lambda_{k}\right)$ and $H_{0}>0$ such that if $H \leq H_{0}$ it is valid that

$$
\begin{aligned}
\left\|u_{k}^{h_{l}}-u_{k}\right\|_{a} & \leq C_{0} \delta_{h_{l}}\left(\lambda_{k}\right), \\
\left\|u_{k}^{h_{l}}-u_{k}\right\|_{-1 / 2, \partial \Omega} & \leq C_{0} C_{1} \sigma\left(h_{l}\right) \delta_{h_{l}}\left(\lambda_{k}\right), \\
\left|\lambda_{k}^{h_{l}}-\lambda_{k}\right| & \leq C_{4} C_{0}^{2} \delta_{h_{l}}^{2}\left(\lambda_{k}\right), \quad l \geq i 0 .
\end{aligned}
$$

Proof. We only prove the result (54) since (53) and (55) can be proved analogously by referring to [16].

The proof is completed by using induction, Theorems 7 and 8 . Note that $\delta_{H}\left(\lambda_{k}\right) \leq \lambda_{k} \sigma(H) \rightarrow 0(H \rightarrow 0)$; then there exists a proper small $H_{0}>0$ such that if $H \leq H_{0}$, Lemma 3 and the following inequalities hold:

$$
\begin{array}{r}
C_{0} \delta_{H}\left(\lambda_{k}\right) \leq \delta_{0}, \\
C_{4} C_{0}^{2} \delta_{H}^{2}\left(\lambda_{k}\right) \leq \delta_{0}, \\
\delta_{H}\left(\lambda_{j}\right) \leq \delta_{0}, \\
\sigma(H) \leq \delta_{0}, \\
C_{4}^{2} C_{0}^{4} \delta_{H}^{2}\left(\lambda_{k}\right) \lambda_{k} \frac{1}{\beta_{0}^{\prime}} \frac{1}{\beta_{0}}+C_{4} C_{0}^{3} C_{1} \delta_{H}^{2}\left(\lambda_{k}\right) \frac{1}{\beta_{0}^{\prime}} \frac{1}{\beta_{0}} \leq 1,
\end{array}
$$

where $j=k-1, k, k+q(j \neq 0)$.

When $l=i 0$, it is easy to know that (53)-(55) are valid (see $[12,16])$. Suppose that Theorem 10 holds for $l-1$; that is, there exists $\bar{u} \in M\left(\lambda_{k}\right)$ such that

$$
\begin{aligned}
\left\|u_{k}^{h_{l-1}}-\bar{u}\right\|_{a} & \leq C_{0} \delta_{h_{l-1}}\left(\lambda_{k}\right), \\
\left\|u_{k}^{h_{l-1}}-\bar{u}\right\|_{-1 / 2, \partial \Omega} & \leq C_{0} C_{1} \sigma\left(h_{l-1}\right) \delta_{h_{l-1}}\left(\lambda_{k}\right), \\
\left|\lambda_{k}^{h_{l-1}}-\lambda_{k}\right| & \leq C_{4} C_{0}^{2} \delta_{h_{l-1}}^{2}\left(\lambda_{k}\right) .
\end{aligned}
$$

Then we infer from (56) that the conditions of Theorem 7 hold.

From Theorems 7 and 8 we get

$$
\begin{aligned}
& \left\|u_{k}^{h_{l}}-u_{k}\right\|_{-1 / 2, \partial \Omega} \leq \frac{C_{0} C_{1}}{2}\left\{\left|\lambda_{0}-\lambda_{k}\right|\right. \\
& \cdot\left(\left|\lambda_{k}^{h_{l-1}}-\lambda_{k}\right|+\left\|u_{k}^{h_{l-1}}-\bar{u}\right\|_{-1 / 2, \partial \Omega}\right)+\sigma\left(h_{l}\right) \\
& \left.\cdot \delta_{h_{l}}\left(\lambda_{k}\right)\right\}, \quad l \geq 1 .
\end{aligned}
$$

Therefore, for $l$, from (59) we derive that

$$
\begin{aligned}
& \left\|u_{k}^{h_{l}}-u_{k}\right\|_{-1 / 2, \partial \Omega} \leq \frac{C_{0} C_{1}}{2}\left\{C_{4}^{2} C_{0}^{4} \delta_{h_{i 0}}^{2}\left(\lambda_{k}\right) \delta_{h_{l-1}}^{2}\left(\lambda_{k}\right)\right. \\
& \quad+C_{4} C_{0}^{3} C_{1} \delta_{h_{i 0}}^{2}\left(\lambda_{k}\right) \sigma\left(h_{l-1}\right) \delta_{h_{l-1}}\left(\lambda_{k}\right) \\
& \left.\quad+\sigma\left(h_{l}\right) \delta_{h_{l}}\left(\lambda_{k}\right)\right\}
\end{aligned}
$$




$$
\begin{aligned}
& \leq \frac{C_{0} C_{1}}{2}\left\{C_{4}^{2} C_{0}^{4} \delta_{h_{i 0}}^{2}\left(\lambda_{k}\right) \lambda_{k} \frac{1}{\beta_{l}^{\prime}} \frac{1}{\beta_{l}}\right. \\
& \left.+C_{4} C_{0}^{3} C_{1} \delta_{h_{i 0}}^{2}\left(\lambda_{k}\right) \frac{1}{\beta_{l}^{\prime}} \frac{1}{\beta_{l}}+1\right\} \sigma\left(h_{l}\right) \delta_{h_{l}}\left(\lambda_{k}\right) \\
& \leq \frac{C_{0} C_{1}}{2}\left\{C_{4}^{2} C_{0}^{4} \delta_{H}^{2}\left(\lambda_{k}\right) \lambda_{k} \frac{1}{\beta_{0}^{\prime}} \frac{1}{\beta_{0}}\right. \\
& \left.+C_{4} C_{0}^{3} C_{1} \delta_{H}^{2}\left(\lambda_{k}\right) \frac{1}{\beta_{0}^{\prime}} \frac{1}{\beta_{0}}+1\right\} \sigma\left(h_{l}\right) \delta_{h_{l}}\left(\lambda_{k}\right),
\end{aligned}
$$

which together with (57) we get (54) immediately.

\section{A Posteriori Error Estimates of the Inverse Iteration with Fixed-Shift}

Based on the work of $[4,12,17-19]$, in this section, we will discuss the a posteriori error estimates of Scheme 4 for the Steklov eigenvalue problem.

Consider the boundary value problem corresponding to (2): find $w \in H^{1}(\Omega)$ such that

$$
a(w, v)=b(f, v), \quad \forall v \in H^{1}(\Omega),
$$

and its finite element approximation states: find $w_{h} \in V_{h}$ such that

$$
a\left(w_{h}, v\right)=b(f, v), \quad \forall v \in V_{h} .
$$

For any element $T \in \pi_{h}$ with diameter $h_{T}$, we denote by $\mathscr{E}_{T}$ the set of edges, and

$$
\mathscr{E}=\bigcup_{T \in \pi_{h}} \mathscr{E}_{T}
$$

We decompose $\mathscr{E}=\mathscr{E}_{\Omega} \cup \mathscr{E}_{\Gamma}$, where $\mathscr{E}_{\Omega}$ and $\mathscr{E}_{\Gamma}$ refer to interior edges and edges on the boundary $\Gamma=\partial \Omega$, respectively. For each $\ell \in \mathscr{E}_{\Omega}$, we choose an arbitrary unit normal vector $n_{\ell}$ and denote the two triangles sharing this edge by $T_{\text {in }}$ and $T_{\text {out }}$, where $n_{\ell}$ points outwards $T_{\text {in }}$.

For $v_{h} \in V_{h}$ we set

$$
\left[\left[\frac{\partial v_{h}}{\partial n_{\ell}}\right]\right]_{\ell}=\nabla\left(\left.v_{h}\right|_{T_{\text {out }}}\right) \cdot n_{\ell}-\nabla\left(\left.v_{h}\right|_{T_{\text {in }}}\right) \cdot n_{\ell} .
$$

Let

$$
\widehat{\lambda}_{k, h_{l}}=\frac{1}{q} \sum_{j=k}^{k+q-1} \lambda_{j, h_{l}}
$$

For each $\ell \in \mathscr{E}$ we define the jump residual:

$$
J_{\ell}\left(u_{k}^{h_{l}}\right)= \begin{cases}\frac{1}{2}\left[\left[\frac{\partial u_{k}^{h_{l}}}{\partial n_{\ell}}\right]\right]_{\ell} & \ell \in \mathscr{E}_{\Omega}, \\ \lambda_{k}^{h_{l}} u_{k}^{h_{l}}-\frac{\partial u_{k}^{h_{l}}}{\partial n_{\ell}} & \ell \in \mathscr{E}_{\Gamma} .\end{cases}
$$

Now, the local error indicator is defined as

$$
\eta_{T}\left(u_{k}^{h_{l}}\right)=\left(h_{T}^{2}\left\|u_{k}^{h_{l}}\right\|_{0, T}^{2}+\sum_{\ell \in \mathscr{E}_{T}}|\ell|\left\|J_{\ell}\left(u_{k}^{h_{l}}\right)\right\|_{0, \ell}^{2}\right)^{1 / 2}
$$

and then the global error estimator is given by

$$
\eta_{\Omega}\left(u_{k}^{h_{l}}\right)=\left(\sum_{T \in \pi_{h}} \eta_{T}^{2}\left(u_{k}^{h_{l}}\right)\right)^{1 / 2}
$$

Substituting $u^{*}$ for $u_{k}^{h_{l}}$, we can get the definitions of $J_{\ell}\left(u^{*}\right)$, $\eta_{T}\left(u^{*}\right)$, and $\eta_{\Omega}\left(u^{*}\right)$ similarly.

Now, we will estimate the error $e=u_{k}-u^{*}$.

From $[4,12]$, we give the following two lemmas among which Lemma 11 provides the global upper bound of $e$, while Lemma 12 provides the local lower bound of $e$.

Lemma 11. The error $e=u_{k}-u^{*}$ satisfies

$$
\|e\|_{a} \leq C_{6}\left\{\eta_{\Omega}\left(u^{*}\right)+\sigma\left(h_{l}\right) \delta_{h_{l}}\left(\lambda_{k}\right)\right\}
$$

Lemma 12. The error $e=u_{k}-u^{*}$ satisfies the following:

(a) For $T \in \pi_{h}$, if $\partial T \cap \Gamma=\emptyset$, then

$$
\eta_{T}\left(u^{*}\right) \leq C_{7}\|e\|_{1, T^{*}},
$$

where $T^{*}$ denotes the union of $T$ and the triangles sharing an edge with $T$.

(b) For $T \in \pi_{h}$, if $\partial T \cap \Gamma \neq \emptyset$, then

$$
\begin{aligned}
& \eta_{T}\left(u^{*}\right) \\
& \quad \leq C_{8}\left\{\|e\|_{1, T}+\sum_{\ell \in \mathscr{E}_{T} \cap \mathscr{E}_{\Gamma}}|\ell|^{1 / 2}\left\|\lambda_{k} u_{k}-\hat{\lambda}_{k, h_{l}} u^{*}\right\|_{0, \ell}\right\} .
\end{aligned}
$$

Next, we will analyze the error $u_{k}^{h_{l}}-u^{*}$.

Theorem 13. Suppose that the conditions of Theorem 10 are satisfied; then

$$
\left\|u_{k}^{h_{l}}-u^{*}\right\|_{a} \leq \frac{C_{0}}{4} \sigma\left(h_{l}\right) \delta_{h_{l}}\left(\lambda_{k}\right)
$$

Proof. Note that $\delta_{H}\left(\lambda_{k}\right) \leq \lambda_{k} \sigma(H) \rightarrow 0(H \rightarrow 0)$; then there exists a proper small $H_{0}>0$ such that if $H \leq H_{0}$, the following inequality holds:

$$
\begin{gathered}
C_{4}^{2} C_{0}^{4} \delta_{\mathrm{H}}^{2}\left(\lambda_{k}\right) \lambda_{k} \frac{1}{\beta_{0}^{\prime}} \frac{1}{\beta_{0}}+C_{4} C_{0}^{3} C_{1} \delta_{H}^{2}\left(\lambda_{k}\right) \frac{1}{\beta_{0}^{\prime}} \frac{1}{\beta_{0}} \\
+C_{4} C_{0}^{2} \delta_{H}^{2}\left(\lambda_{k}\right) \leq 1 .
\end{gathered}
$$

From (44), Theorem 10, and Condition 9, we have

$$
\begin{gathered}
\left\|u_{k}^{h_{l}}-u^{*}\right\|_{a} \leq \frac{C_{0}}{4}\left\{C_{4}^{2} C_{0}^{4} \delta_{h_{i 0}}^{2}\left(\lambda_{k}\right) \delta_{h_{l-1}}^{2}\left(\lambda_{k}\right)\right. \\
+C_{4} C_{0}^{3} C_{1} \delta_{h_{i 0}}^{2}\left(\lambda_{k}\right) \sigma\left(h_{l-1}\right) \delta_{h_{l-1}}\left(\lambda_{k}\right) \\
\left.+C_{4} C_{0}^{2} \delta_{h_{i 0}}^{2}\left(\lambda_{k}\right) \sigma\left(h_{l}\right) \delta_{h_{l}}\left(\lambda_{k}\right)\right\}
\end{gathered}
$$




$$
\begin{aligned}
& \leq \frac{C_{0}}{4}\left\{C_{4}^{2} C_{0}^{4} \delta_{h_{i 0}}^{2}\left(\lambda_{k}\right) \lambda_{k} \frac{1}{\beta_{l}^{\prime}} \frac{1}{\beta_{l}}\right. \\
& \left.+C_{4} C_{0}^{3} C_{1} \delta_{h_{i 0}}^{2}\left(\lambda_{k}\right) \frac{1}{\beta_{l}^{\prime}} \frac{1}{\beta_{l}}+C_{4} C_{0}^{2} \delta_{h_{i 0}}^{2}\left(\lambda_{k}\right)\right\} \sigma\left(h_{l}\right) \\
& \cdot \delta_{h_{l}}\left(\lambda_{k}\right) \leq \frac{C_{0}}{4}\left\{C_{4}^{2} C_{0}^{4} \delta_{H}^{2}\left(\lambda_{k}\right) \lambda_{k} \frac{1}{\beta_{0}^{\prime}} \frac{1}{\beta_{0}}\right. \\
& \left.+C_{4} C_{0}^{3} C_{1} \delta_{H}^{2}\left(\lambda_{k}\right) \frac{1}{\beta_{0}^{\prime}} \frac{1}{\beta_{0}}+C_{4} C_{0}^{2} \delta_{H}^{2}\left(\lambda_{k}\right)\right\} \sigma\left(h_{l}\right) \\
& \cdot \delta_{h_{l}}\left(\lambda_{k}\right),
\end{aligned}
$$

which together with (73) yields (72) immediately.

We give the following lemma by referring to [12] (see Lemma 3.4 in [12]).

Lemma 14. Suppose that the conditions of Theorem 10 are satisfied; then

$$
\begin{aligned}
& \left|\eta_{T}\left(u^{*}\right)-\eta_{T}\left(u_{k}^{h_{l}}\right)\right| \\
& \quad \leq C_{9}\left\{\delta_{h_{l}}^{2}\left(\lambda_{k}\right)\left\|u^{*}\right\|_{1, T}+\left\|u_{k}^{h_{l}}-u^{*}\right\|_{1, T}\right\}, \\
& \left|\eta_{\Omega}\left(u^{*}\right)-\eta_{\Omega}\left(u_{k}^{h_{l}}\right)\right| \\
& \quad \leq C_{10}\left\{\delta_{h_{l}}^{2}\left(\lambda_{k}\right)\left\|u^{*}\right\|_{a}+\left\|u_{k}^{h_{l}}-u^{*}\right\|_{a}\right\} .
\end{aligned}
$$

In the following discussion, combining Lemmas 11, 12, and 14 and Theorem 13, we give the global upper bound and the local lower bound of the error.

Theorem 15. Suppose that the conditions of Theorem 10 are satisfied; then there exists $u_{k} \in M\left(\lambda_{k}\right)$ such that

$$
\left\|u_{k}-u_{k}^{h_{l}}\right\|_{a} \leq C_{6} \eta_{\Omega}\left(u_{k}^{h_{l}}\right)+R_{1}
$$

where $R_{1}=C_{6} C_{10} \delta_{h_{l}}^{2}\left(\lambda_{k}\right)\left\|u^{*}\right\|_{a}+\left(C_{6}+C_{0} / 4+C_{6} C_{10}\left(C_{0} /\right.\right.$ 4)) $\sigma\left(h_{l}\right) \delta_{h_{l}}\left(\lambda_{k}\right)$.

Proof. Select $u_{k} \in M\left(\lambda_{k}\right)$ which is given by (41); then from Lemma 11, Theorem 13, and (76) we get

$$
\begin{aligned}
& \left\|u_{k}-u_{k}^{h_{l}}\right\|_{a} \leq\left\|u_{k}-u^{*}\right\|_{a}+\left\|u^{*}-u_{k}^{h_{l}}\right\|_{a} \leq C_{6}\left\{\eta_{\Omega}\left(u^{*}\right)\right. \\
& \left.+\sigma\left(h_{l}\right) \delta_{h_{l}}\left(\lambda_{k}\right)\right\}+\frac{C_{0}}{4} \sigma\left(h_{l}\right) \delta_{h_{l}}\left(\lambda_{k}\right) \\
& \leq C_{6}\left\{\eta_{\Omega}\left(u_{k}^{h_{l}}\right)\right. \\
& +C_{10}\left(\delta_{h_{l}}^{2}\left(\lambda_{k}\right)\left\|u^{*}\right\|_{a}+\left\|u_{k}^{h_{l}}-u^{*}\right\|_{a}\right) \\
& \left.+\sigma\left(h_{l}\right) \delta_{h_{l}}\left(\lambda_{k}\right)\right\}+\frac{C_{0}}{4} \sigma\left(h_{l}\right) \delta_{h_{l}}\left(\lambda_{k}\right) \\
& \leq C_{6} \eta_{\Omega}\left(u_{k}^{h_{l}}\right)+R_{1} .
\end{aligned}
$$

The proof is completed.
It is obvious that $R_{1}$ is a higher order term. Hence, we obtain that $\eta_{\Omega}\left(u_{k}^{h_{l}}\right)$ is a global reliable error indicator of $\| u_{k}-$ $u_{k}^{h_{l}} \|_{a}$.

Theorem 16. Under the conditions of Theorem 10, there exists $u_{k} \in M\left(\lambda_{k}\right)$ such that the following hold:

(a) For $T \in \pi_{h_{l}}$, if $\partial T \cap \Gamma=\emptyset$, then

$$
\eta_{T}\left(u_{k}^{h_{l}}\right) \leq C_{7}\left\|u_{k}-u_{k}^{h_{l}}\right\|_{1, T^{*}}+R_{2},
$$

where $R_{2}=\left(C_{7}+C_{9}\right)\left\|u_{k}^{h_{l}}-u^{*}\right\|_{1, T^{*}}+C_{9} \delta_{h_{l}}^{2}\left(\lambda_{k}\right)\left\|u^{*}\right\|_{1, T}$.

(b) For $T \in \pi_{h_{l}}$, if $\partial T \cap \Gamma \neq \emptyset$, then

$$
\eta_{T}\left(u_{k}^{h_{l}}\right) \leq C_{8}\left\|u_{k}-u_{k}^{h_{l}}\right\|_{1, T}+R_{3},
$$

where

$$
\begin{aligned}
R_{3}= & \left(C_{8}+C_{9}\right)\left\|u_{k}^{h_{l}}-u^{*}\right\|_{1, T}+C_{9} \delta_{h_{l}}^{2}\left(\lambda_{k}\right)\left\|u^{*}\right\|_{1, T} \\
& +C_{8} \sum_{\ell \in \mathscr{C}_{T} \cap \mathscr{E}_{\Gamma}}|\ell|^{1 / 2}\left\|\lambda_{k} u_{k}-\widehat{\lambda}_{k, h_{l}} u^{*}\right\|_{0, \ell} .
\end{aligned}
$$

Proof. We can prove the desired results by using the proof method of Theorem 3.4 in [12].

According to Remark 3.1 in [4] and Remark 3.2 in [12] we know that the term $\sum_{\ell \in \mathscr{E}_{T} \cap \mathscr{E}_{\Gamma}}|\ell|^{1 / 2}\left\|\lambda_{k} u_{k}-\widehat{\lambda}_{k, h_{l}} u^{*}\right\|_{0, \ell}$ is a higher order term. From Theorem 13, we know that $\| u_{k}^{h_{l}}-$ $u^{*} \|_{1, T^{*}}$ and $\left\|u_{k}^{h_{l}}-u^{*}\right\|_{1, T}$ are also higher order terms. And it is obvious that $\delta_{h_{l}}^{2}\left(\lambda_{k}\right)\left\|u^{*}\right\|_{1, T}$ is a higher order term. Therefore, from (79) and (80) we know that $\eta_{T}\left(u_{k}^{h_{l}}\right)$ is an efficient local error indicator of $\left\|u_{k}-u_{k}^{h_{l}}\right\|_{1, T^{*}}$ and $\left\|u_{k}-u_{k}^{h_{l}}\right\|_{1, T}$.

In the following theorem, we give the estimate for approximate eigenvalue.

Theorem 17. Suppose that the conditions of Theorem 10 are satisfied; then

$$
\left|\lambda_{k}^{h_{l}}-\lambda_{k}\right|=\mathcal{O}\left(\eta_{\Omega}^{2}\left(u_{k}^{h_{l}}\right)\right)
$$

Proof. From Theorem 10 and Lemma 1 it is easy to prove that

$$
\lambda_{k}^{h_{l}}-\lambda_{k}=\mathcal{O}\left(\left\|u_{k}^{h_{l}}-u_{k}\right\|_{a}^{2}\right)
$$

then combining with Theorem 16 and (77), we can get the desired result (82).

\section{Numerical Experiments}

In this section we first give an adaptive algorithm of the Rayleigh quotient iteration type and establish an adaptive algorithm of fixed-shift inverse iteration type for the Steklov eigenvalue problem.

The following Algorithm 1 of the Rayleigh quotient iteration type refers to Algorithm 4.3 in [12] or Algorithm 6.1 in [16]. 
TABLE 1: The 1st and the 2nd eigenvalues of Example 1 obtained by Algorithms 1 and 2 with $H=\sqrt{2} / 32$.

\begin{tabular}{cccccccc}
\hline$k$ & $l$ & $N_{k, l}(1)$ & $\lambda_{k}^{h_{l}}(1)$ & $\mathrm{CPU}_{k, l}^{(1)}$ & $N_{k, l}(2)$ & $\lambda_{k}^{h_{l}}(2)$ & $\mathrm{CPU}_{k, l}^{(2)}$ \\
\hline 1 & 5 & 5700 & 0.24008040 & 0.54 & 5700 & 0.24008040 & 0.57 \\
1 & 10 & 27344 & 0.24007936 & 1.60 & 27343 & 0.24007936 & 0.24007915 \\
1 & 15 & 117001 & 0.24007915 & 7.67 & 117001 & 0.24007910 \\
1 & 19 & 408971 & 0.24007910 & 29.06 & 408971 & 29.25 \\
1 & 20 & 509032 & 0.24007910 & 39.88 & 509032 & 0.24007910 & 40.07 \\
1 & 21 & 764069 & 0.24007909 & 55.90 & 764069 & 0.24007909 & 55.96 \\
2 & 5 & 4526 & 1.49245505 & 0.51 & 4529 & 1.49245448 & 0.54 \\
2 & 12 & 37855 & 1.49231870 & 2.31 & 37941 & 1.49231871 & 2.33 \\
2 & 18 & 223337 & 1.49230601 & 15.77 & 224277 & 1.49230600 & 15.89 \\
2 & 24 & 1329617 & 1.49230362 & 109.68 & 1334272 & 1.49230362 & 109.90 \\
2 & 25 & 1805637 & 1.49230347 & 151.36 & 1812726 & 1.49230347 & 151.02 \\
2 & 26 & 2439573 & 1.49230337 & 211.29 & 2447212 & 1.49230337 \\
\hline
\end{tabular}

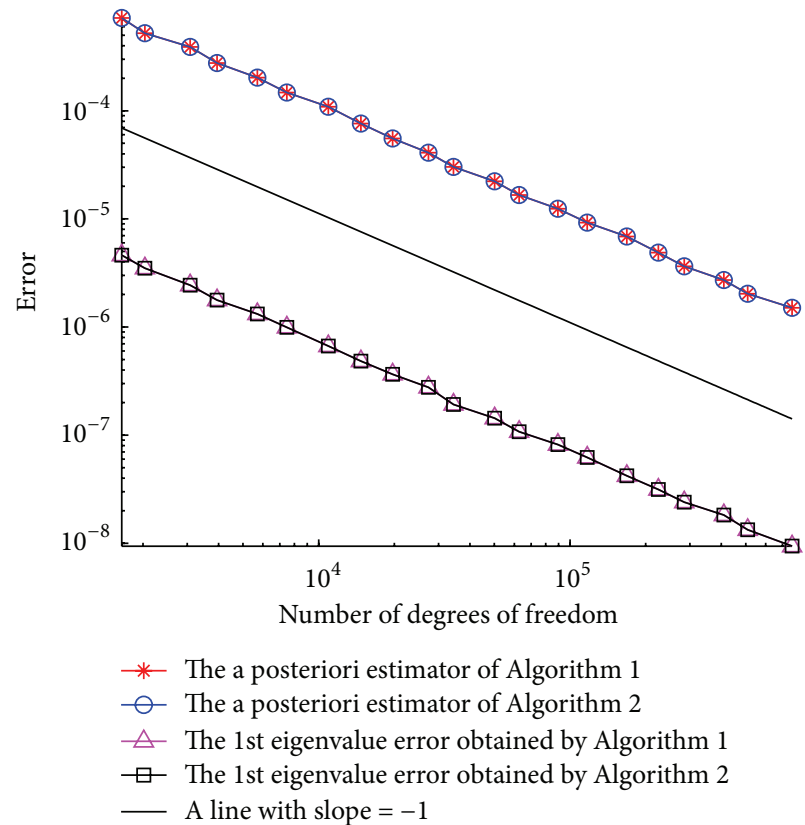

(a)

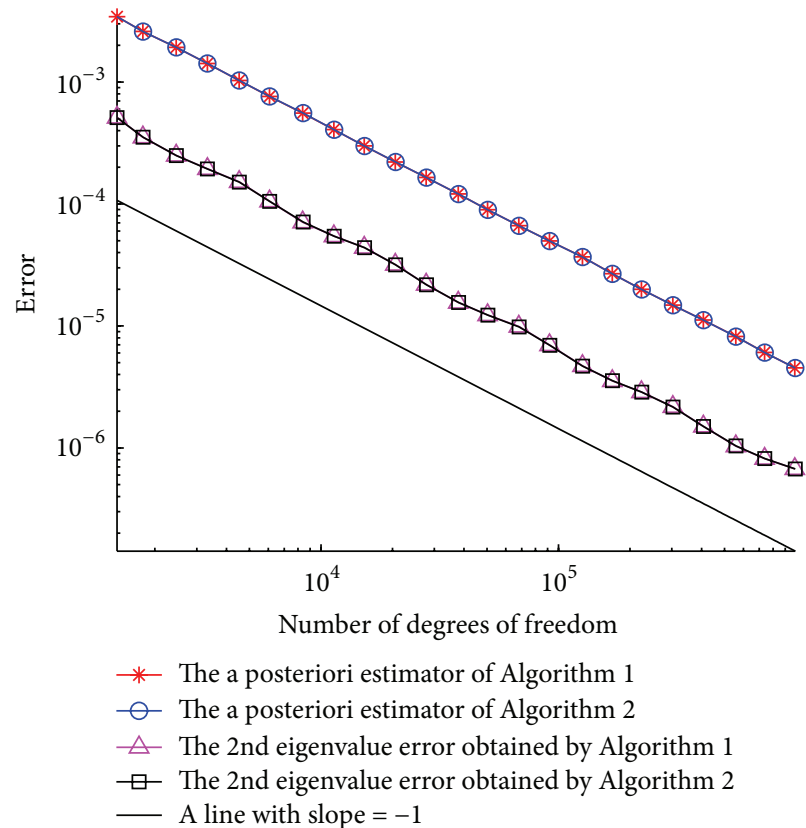

(b)

FIgure 1: The curves of error and the a posteriori error estimators of two algorithms for the 1st (a) and 2nd (b) eigenvalues on the square domain.

Algorithm 1. Choose parameter $0<\omega<1$.

Step 1. Pick any initial mesh $\pi_{h_{0}}$.

Step 2. Solve (2) on $\pi_{h_{0}}$ for discrete solution $\left(\lambda^{h_{0}}, u^{h_{0}}\right)$.

Step 3. Let $l \Leftarrow 0, \lambda_{0} \Leftarrow \lambda^{h_{0}}$.

Step 4. Compute the local indicators $\eta_{T}\left(u^{h_{l}}\right)$.

Step 5. Construct $\widehat{\pi}_{h_{l}} \subset \pi_{h_{l}}$ by Marking Strategy E and $\omega$.

Step 6. Refine $\pi_{h_{l}}$ to get a new mesh $\pi_{h_{l+1}}$ by Procedure REFINE.
Step 7. Find $u^{\prime} \in V_{h_{l+1}}$ such that

$$
a\left(u^{\prime}, v\right)-\lambda_{0} b\left(u^{\prime}, v\right)=b\left(u^{h_{l}}, v\right), \quad \forall v \in V_{h_{l+1}}
$$

denote $u^{h_{l+1}}=u^{\prime} /\left\|u^{\prime}\right\|_{a}$ and compute the Rayleigh quotient

$$
\lambda^{h_{l+1}}=\frac{a\left(u^{h_{l+1}}, u^{h_{l+1}}\right)}{b\left(u^{h_{l+1}}, u^{h_{l+1}}\right)} .
$$

Step 8. Let $\lambda_{0} \Leftarrow \lambda^{h_{l+1}}, l \Leftarrow l+1$ and go to Step 4 .

Marking Strategy E. Give parameter $0<\omega<1$. 
TABLE 2: The 1st and the 3rd eigenvalues of Example 2 obtained by two algorithms with $H=\sqrt{2} / 32$.

\begin{tabular}{cccccccc}
\hline$k$ & $l$ & $N_{k, l}(1)$ & $\lambda_{k}^{h_{l}}(1)$ & $\mathrm{CPU}_{k, l}^{(1)}$ & $N_{k, l}(2)$ & $\lambda_{k}^{h_{l}}(2)$ & 0.18296573 \\
\hline 1 & 5 & 4369 & 0.18296573 & 0.46 & 4369 & 0.45 \\
1 & 12 & 35119 & 0.18296446 & 2.00 & 35119 & 0.18296446 \\
1 & 18 & 227719 & 0.18296426 & 14.90 & 227719 & 0.18296426 \\
1 & 21 & 557370 & 0.18296425 & 39.77 & 557370 & 0.18296425 \\
1 & 22 & 738108 & 0.18296425 & 55.04 & 738108 & 0.18296425 \\
1 & 23 & 1105016 & 0.18296424 & 78.27 & 1105016 & 0.18296424 \\
3 & 5 & 3690 & 1.68889444 & 0.49 & 3690 & 1.68889444 \\
3 & 12 & 31089 & 1.68863207 & 1.79 & 31089 & 1.68863207 \\
3 & 18 & 183089 & 1.68860633 & 11.84 & 183089 & 1.68860633 \\
3 & 24 & 1098418 & 1.68860134 & 81.42 & 1098418 & 1.68860134 \\
3 & 25 & 1485695 & 1.68860109 & 112.52 & 1485695 & 1.683 \\
3 & 26 & 2002315 & 1.68860097 & 155.64 & 2002315 & 1.83 \\
\hline
\end{tabular}

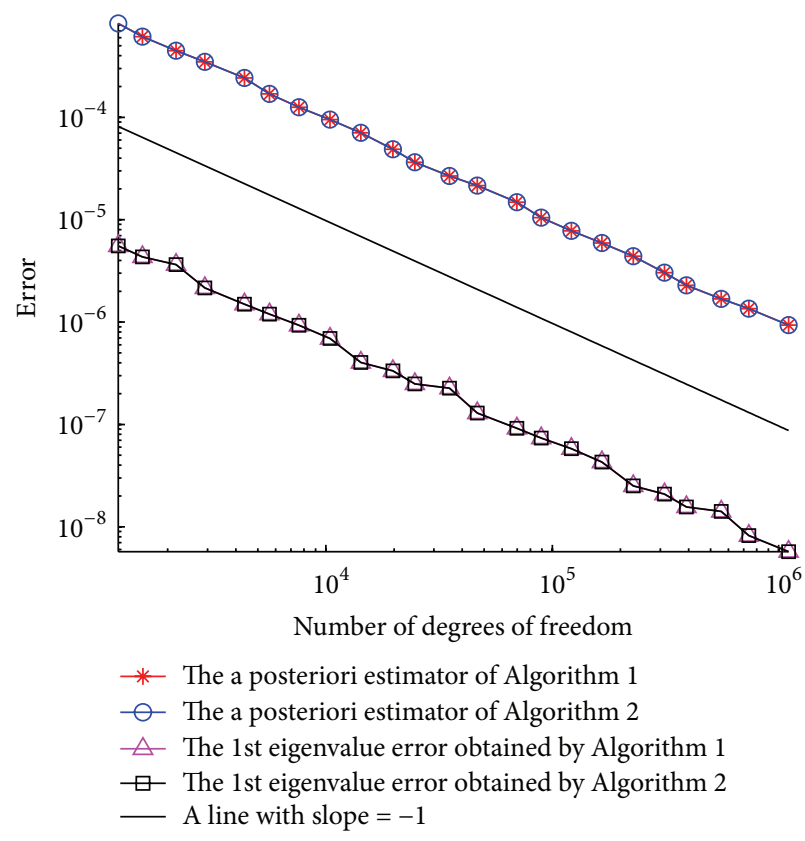

(a)

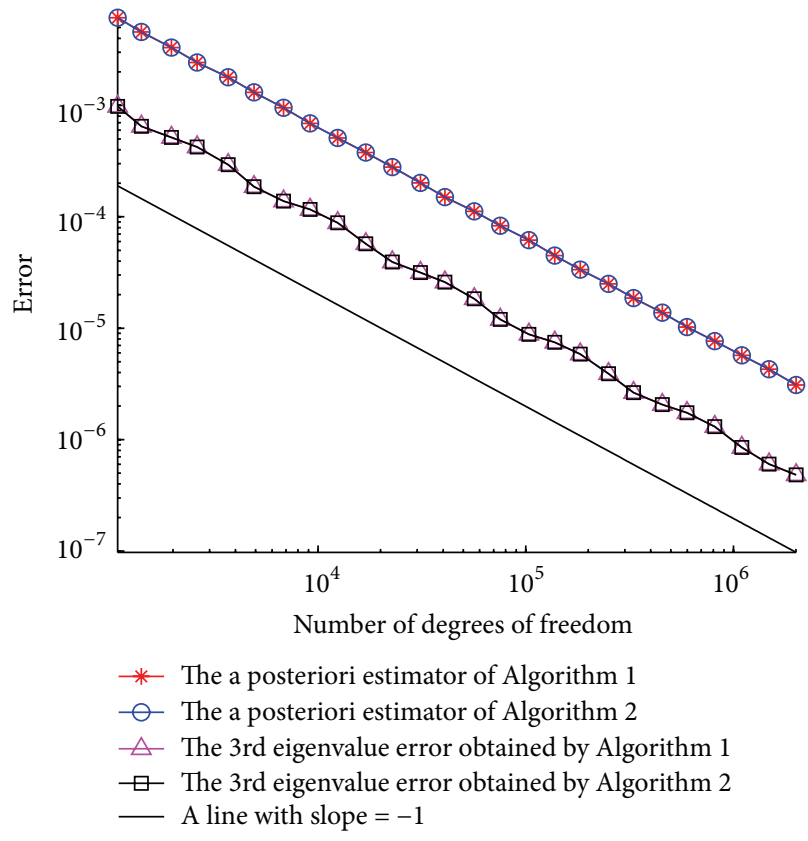

(b)

FIgURE 2: The curves of error and the a posteriori error estimators of two algorithms for the 1st (a) and 3rd (b) eigenvalues on the $L$-shaped domain.

Step 1. Construct a minimal subset $\widehat{\pi}_{h_{l}}$ of $\pi_{h_{l}}$ by selecting some elements in $\pi_{h_{l}}$ such that

$$
\sum_{T \in \hat{\pi}_{h_{l}}} \eta_{T}^{2}\left(u^{h_{l}}\right) \geq \omega \eta_{\Omega}^{2}\left(u^{h_{l}}\right) .
$$

Step 2. Mark all the elements in $\widehat{\pi}_{h_{l}}$.

$\eta_{T}\left(u^{h_{l}}\right)$ and $\eta_{\Omega}\left(u^{h_{l}}\right)$ are defined by (67) and (68) with $u_{k}^{h_{l}}$ and $\lambda_{k}^{h_{l}}$ replaced by $u^{h_{l}}$ and $\lambda^{h_{l}}$, respectively.

Note that when $\left|\lambda_{0}-\lambda\right|$ is too small, (84) is an almost singular linear equation. Although it has no difficulty in solving (84) numerically (see [12]), one would like to think of selecting a proper integer $i 0 \geq 0$ to establish the following adaptive algorithm.
Algorithm 2. Choose parameter $0<\omega<1$.

Steps 1-7. Execute Steps 1-7 of Algorithm 1.

Step 8. If $l<i_{0}, \lambda_{0} \Leftarrow \lambda^{h_{l+1}}, l \Leftarrow l+1$, go to Step 4; else $l \Leftarrow l+1$, go to Step 4 .

Marking Strategy E in Algorithm 2 is the same as that in Algorithm 1.

Now, we will implement some numerical experiments to validate our theoretical analysis and show the efficiency of Algorithm 2 with $i_{0}=0$. We use MATLAB 2012 together with the package of Chen [20] to solve Examples 1, 2, and 3, and we take $\omega=0.5$. 
TABLE 3: The 1st and 5th eigenvalues of Example 3 obtained by two algorithms with $H=\sqrt{2} / 32$.

\begin{tabular}{|c|c|c|c|c|c|c|c|}
\hline$k$ & $l$ & $N_{k, l}(1)$ & $\lambda_{k}^{h_{l}}(1)$ & $\mathrm{CPU}_{k, l}^{(1)}$ & $N_{k, l}(2)$ & $\lambda_{k}^{h_{l}}(2)$ & $\mathrm{CPU}_{k, l}^{(2)}$ \\
\hline 1 & 5 & 9391 & 0.23957586 & 0.66 & 9391 & 0.23957586 & 0.75 \\
\hline 1 & 10 & 42645 & 0.23957397 & 2.45 & 42645 & 0.23957397 & 2.56 \\
\hline 1 & 15 & 189550 & 0.23957352 & 12.52 & 189550 & 0.23957352 & 12.72 \\
\hline 1 & 19 & 634556 & 0.23957342 & 48.00 & 634556 & 0.23957342 & 47.09 \\
\hline 1 & 20 & 860490 & 0.23957341 & 66.32 & 860490 & 0.23957341 & 65.38 \\
\hline 1 & 21 & 1131274 & 0.23957340 & 91.04 & 1131274 & 0.23957340 & 90.05 \\
\hline 5 & 5 & 9842 & 1.41254843 & 0.70 & 9842 & 1.41254843 & 0.74 \\
\hline 5 & 12 & 77005 & 1.41241115 & 4.53 & 77005 & 1.41241115 & 4.68 \\
\hline 5 & 18 & 453543 & 1.41238410 & 31.53 & 453543 & 1.41238410 & 31.74 \\
\hline 5 & 21 & 1092642 & 1.41238245 & 82.23 & 1092642 & 1.41238245 & 82.74 \\
\hline 5 & 22 & 1497488 & 1.41238165 & 112.92 & 1497488 & 1.41238165 & 113.82 \\
\hline 5 & 23 & 1993327 & 1.41238104 & 155.36 & 1993327 & 1.41238104 & 156.14 \\
\hline
\end{tabular}

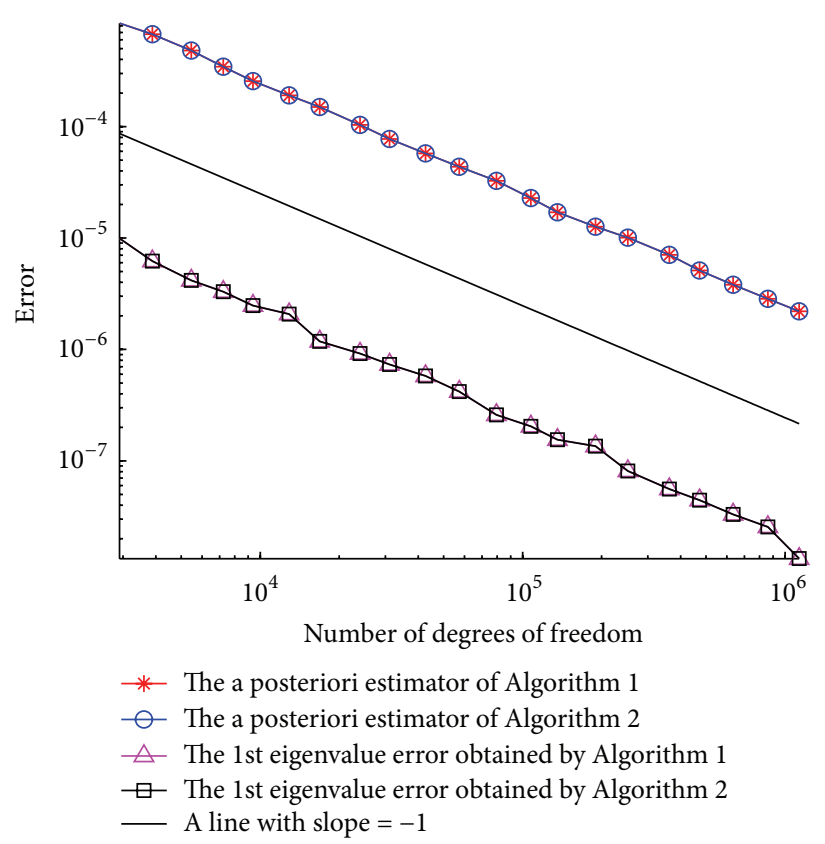

(a)

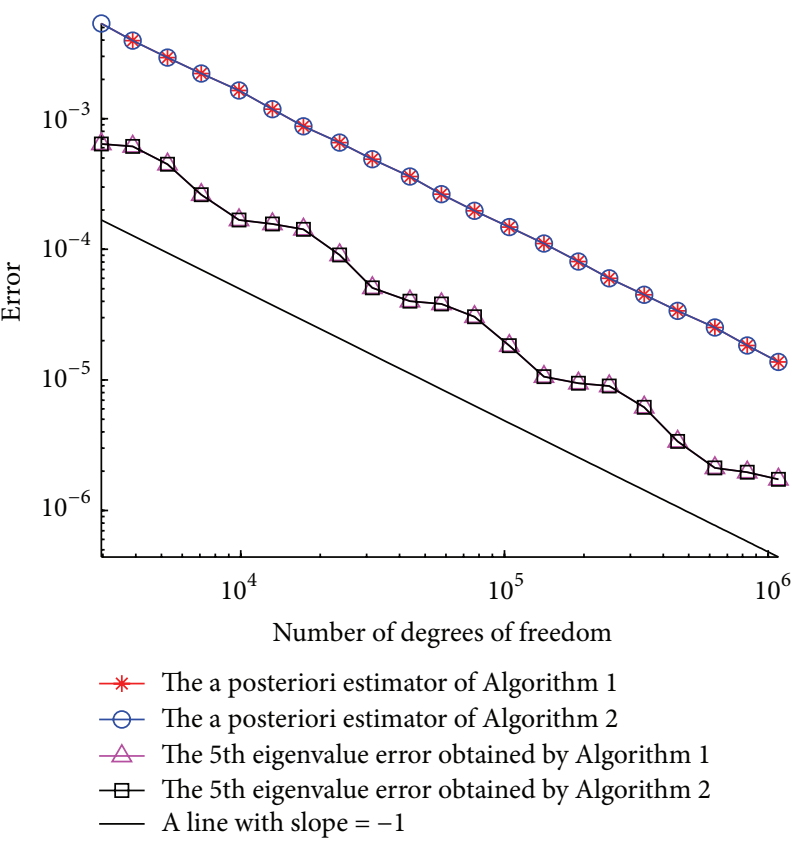

(b)

FIgURE 3: The curves of error and the a posteriori error estimators of two algorithms for the 1st (a) and 5th (b) eigenvalues on slit domain.

For reading conveniently, we use the following notations in our tables:

$\lambda_{k}^{h_{l}}(m)$ : the $k$ th eigenvalue derived from the $l$ th iteration obtained by Algorithm $m(m=1,2)$.

$\left|\lambda_{k}^{h_{l}}(m)-\lambda_{k}\right|:$ the error of $\lambda_{k}^{h_{l}}(m)$ obtained by Algorithm $m(m=1,2)$.

$N_{k, l}(m)$ : the degrees of freedom of the $l$ th iteration for $\lambda_{k}^{h_{l}}(m)(m=1,2)$.

$\mathrm{CPU}_{k, l}^{(m)}(\mathrm{s})$ : the CPU time(s) from the program starting to calculate result of the $l$ th iteration appearing by using Algorithm $m(m=1,2)$.

Example 1. We use Algorithms 1 and 2 to compute the approximations of the 1st and the 2nd eigenvalue of (1) with the triangle linear finite element on $\Omega=[0,1] \times[0,1]$. The numerical results are listed in Table 1.

Since the exact eigenvalues are unknown, we use $\lambda_{1} \approx$ 0.24007908542 and $\lambda_{2} \approx 1.49230313453$ obtained by the spectral element method (see [21]) as the reference eigenvalues. We show the error curves and the a posteriori estimators obtained by two algorithms for $\lambda_{1}$ and $\lambda_{2}$ in Figure 1. It can be seen from Figure 1 that the error curves are approximately parallel to the line with slope -1 , which indicates that Algorithm 2 achieves the optimal convergence rate of $\mathcal{O}\left(N_{l}^{-1}\right)$ as well as Algorithm 1.

Observing the numerical results in Table 1, we can find that when the degrees of freedom are almost the same, the approximate eigenvalues obtained by Algorithm 2 are nearly as accurate as those obtained by Algorithm 1 and their CPU time are roughly the same. 
TABLE 4: The results of Example 4 on $\Omega=[0,1] \times[0,1]$.

\begin{tabular}{|c|c|c|c|c|c|c|}
\hline$k$ & $l$ & $N_{k, l}$ & $\lambda_{k}^{h_{l}}(3)$ & $\mathrm{CPU}_{k, l}^{(3)}$ & $\lambda_{k}^{h_{l}}(2)$ & $\mathrm{CPU}_{k, l}^{(2)}$ \\
\hline 1 & 1 & 1089 & 0.240088481 & 0.08 & 0.240088481 & 0.18 \\
\hline 1 & 2 & 4225 & 0.240081438 & 0.17 & 0.240081438 & 0.22 \\
\hline 1 & 3 & 16641 & 0.240079674 & 0.48 & 0.240079674 & 0.43 \\
\hline 1 & 4 & 66049 & 0.240079233 & 1.92 & 0.240079233 & 1.45 \\
\hline 1 & 5 & 263169 & 0.240079122 & 8.92 & 0.240079122 & 6.48 \\
\hline 1 & 6 & 1050625 & 0.240079095 & 38.36 & 0.240079095 & 32.00 \\
\hline 2 & 1 & 1089 & 1.492905398 & 0.11 & 1.492905378 & 0.19 \\
\hline 2 & 2 & 4225 & 1.492454269 & 0.23 & 1.492454267 & 0.24 \\
\hline 2 & 3 & 16641 & 1.492340958 & 0.65 & 1.492340958 & 0.46 \\
\hline 2 & 4 & 66049 & 1.492312593 & 2.67 & 1.492312593 & 1.47 \\
\hline 2 & 5 & 263169 & 1.492305499 & 12.19 & 1.492305499 & 6.57 \\
\hline 2 & 6 & 1050625 & 1.492303726 & 56.05 & 1.492303726 & 32.00 \\
\hline
\end{tabular}

TABLE 5: The results of Example 4 on $\Omega=([0,1] \times[0,1 / 2]) \cup([0,1 / 2] \times[1 / 2,1])$.

\begin{tabular}{|c|c|c|c|c|c|c|}
\hline$k$ & $l$ & $N_{k, l}$ & $\lambda_{k}^{h_{l}}(3)$ & $\mathrm{CPU}_{k, l}^{(3)}$ & $\lambda_{k}^{h_{l}}(2)$ & $\mathrm{CPU}_{k, l}^{(2)}$ \\
\hline 1 & 1 & 833 & 0.182975157 & 0.04 & 0.182975157 & 0.19 \\
\hline 1 & 2 & 3201 & 0.182966980 & 0.11 & 0.182966980 & 0.22 \\
\hline 1 & 3 & 12545 & 0.182964924 & 0.31 & 0.182964924 & 0.36 \\
\hline 1 & 4 & 49665 & 0.182964409 & 1.25 & 0.182964409 & 1.06 \\
\hline 1 & 5 & 197633 & 0.182964280 & 5.88 & 0.182964280 & 4.60 \\
\hline 1 & 6 & 788481 & 0.182964248 & 26.02 & 0.182964248 & 21.61 \\
\hline 3 & 1 & 833 & 1.690165085 & 0.05 & 1.690165013 & 0.20 \\
\hline 3 & 2 & 3201 & 1.688996545 & 0.12 & 1.688996536 & 0.22 \\
\hline 3 & 3 & 12545 & 1.688700132 & 0.34 & 1.688700131 & 0.36 \\
\hline 3 & 4 & 49665 & 1.688625481 & 1.27 & 1.688625481 & 1.04 \\
\hline 3 & 5 & 197633 & 1.688606742 & 5.95 & 1.688606742 & 4.34 \\
\hline 3 & 6 & 788481 & 1.688602046 & 26.11 & 1.688602046 & 20.20 \\
\hline
\end{tabular}

Example 2. We use Algorithms 1 and 2 to compute the approximations of the 1st and the 3 rd eigenvalue of (1) with the triangle linear finite element on $\Omega=([0,1] \times[0,1 / 2]) \cup$ $([0,1 / 2] \times[1 / 2,1])$. The numerical results are presented in Table 2.

In Figure 2 we depict the error curves and the a posteriori estimators obtained by two algorithms for $\lambda_{1}$ and $\lambda_{3}$. Here we use $\lambda_{1} \approx 0.18296423687$ and $\lambda_{3} \approx 1.68860048358$ obtained by the spectral element method (see [21]) as the reference eigenvalues. It can be seen from Figure 2 that the error curves are approximately parallel to the line with slope -1 , which indicates that Algorithm 2 achieves the optimal convergence rate of $\mathcal{O}\left(N_{l}^{-1}\right)$ as well as Algorithm 1.

It also can be seen from Table 2 that when the degrees of freedom are the same, one can use Algorithms 1 and 2 to get the same accurate approximations with nearly the same CPU time.

Example 3. We use Algorithms 1 and 2 to compute the approximations of the 1st and the 5th eigenvalue of (1) with the triangle linear finite element on $\Omega=\left\{\left(x_{1}, x_{2}\right):\left|x_{1}\right|+\right.$ $\left.\left|x_{2}\right|<1\right\} \backslash\left\{\left(x_{1}, x_{2}\right): 0 \leq x_{1} \leq 1, x_{2}=0\right\}$. The numerical results are presented in Table 3 .

Since the exact eigenvalues are unknown, we compute the approximations of two exact eigenvalues of (1): $\lambda_{1} \approx$ 0.23957338768 and $\lambda_{5} \approx 1.41238071918$ by the standard adaptive algorithm (see, e.g., [22]) with the degrees of freedom of more than 5000000 . We show the curves of the error and the a posteriori estimators obtained by two algorithms for $\lambda_{1}$ and $\lambda_{5}$ in Figure 3. We can see from Figure 3 that the error curves are approximately parallel to the line with slope -1 , which indicates that Algorithm 2 achieves the optimal convergence rate of $\mathcal{O}\left(N_{l}^{-1}\right)$ as well as Algorithm 1.

From the numerical results in Table 3, we can conclude that Algorithm 2 is also an efficient approach like Algorithm 1 for solving the Steklov eigenvalue problem.

Example 4. We use the method in [10] (see Algorithms 4.1 and 7.2 there) to compute the numerical eigenvalues of (1) on $[0,1] \times[0,1],([0,1] \times[0,1 / 2]) \cup([0,1 / 2] \times[1 / 2,1])$, and $\left\{\left(x_{1}, x_{2}\right):\left|x_{1}\right|+\left|x_{2}\right|<1\right\} \backslash\left\{\left(x_{1}, x_{2}\right): 0 \leq x_{1} \leq 1, x_{2}=0\right\}$, respectively, and list the associated results in Tables $4-6$ which are denoted by $\lambda_{k}^{h_{l}}(3)$ and $\mathrm{CPU}_{k, l}^{(3)}$. 
TABLE 6: The results of Example 4 on $\Omega=\left\{\left(x_{1}, x_{2}\right):\left|x_{1}\right|+\left|x_{2}\right|<1\right\} \backslash\left\{\left(x_{1}, x_{2}\right): 0 \leq x_{1} \leq 1, x_{2}=0\right\}$.

\begin{tabular}{|c|c|c|c|c|c|c|}
\hline$k$ & $l$ & $N_{k, l}$ & $\lambda_{k}^{h_{l}}(3)$ & $\mathrm{CPU}_{k, l}^{(3)}$ & $\lambda_{k}^{h_{l}}(2)$ & $\mathrm{CPU}_{k, l}^{(2)}$ \\
\hline 1 & 1 & 2145 & 0.239589697 & 0.12 & 0.239589697 & 0.22 \\
\hline 1 & 2 & 8385 & 0.239577621 & 0.28 & 0.239577621 & 0.31 \\
\hline 1 & 3 & 33153 & 0.239574482 & 0.96 & 0.239574482 & 0.77 \\
\hline 1 & 4 & 131841 & 0.239573668 & 4.03 & 0.239573668 & 2.99 \\
\hline 1 & 5 & 525825 & 0.239573457 & 17.43 & 0.239573457 & 13.80 \\
\hline 1 & 6 & 2100225 & 0.239573403 & 78.31 & 0.239573403 & 71.35 \\
\hline 5 & 1 & 2145 & 1.413086665 & 0.12 & 1.413086553 & 0.20 \\
\hline 5 & 2 & 8385 & 1.412557485 & 0.28 & 1.412557472 & 0.29 \\
\hline 5 & 3 & 33153 & 1.412424609 & 0.91 & 1.412424608 & 0.68 \\
\hline 5 & 4 & 131841 & 1.412391332 & 3.92 & 1.412391332 & 2.77 \\
\hline 5 & 5 & 525825 & 1.412383004 & 17.35 & 1.412383004 & 12.79 \\
\hline 5 & 6 & 2100225 & 1.412380921 & 79.20 & 1.412380921 & 64.99 \\
\hline
\end{tabular}

From Tables 4-6 we can see that, with the same degrees of freedom $N_{k, l}$, our method uses less CPU time to obtain the same accurate approximations, especially for multiple eigenvalue $\lambda_{2}$ on $[0,1] \times[0,1]$, comparing with the one in [10].

\section{Competing Interests}

The authors declare that there are no competing interests regarding the publication of this paper.

\section{Acknowledgments}

This work was supported by the National Natural Science Foundation of China (no. 11201093) and the Science and Technology Foundation of Guizhou Province of China (LKS[2013]06).

\section{References}

[1] S. Bergman and M. Schiffer, Kernel Functions and Elliptic Differential Equations in Mathematical Physics, Academic Press, New York, NY, USA, 1953.

[2] C. Conca, J. Planchard, and M. Vanninathan, Fluids and Periodic Structures, vol. 38, John Wiley \& Sons, Chichester, UK, 1995.

[3] A. Bermúdez, R. Rodríguez, and D. Santamarina, "A finite element solution of an added mass formulation for coupled fluid-solid vibrations," Numerische Mathematik, vol. 87, no. 2, pp. 201-227, 2000.

[4] M. G. Armentano and C. Padra, "A posteriori error estimates for the Steklov eigenvalue problem," Applied Numerical Mathematics, vol. 58, no. 5, pp. 593-601, 2008.

[5] A. Alonso and A. Dello Russo, "Spectral approximation of variationally-posed eigenvalue problems by nonconforming methods," Journal of Computational and Applied Mathematics, vol. 223, no. 1, pp. 177-197, 2009.

[6] Q. Li, Q. Lin, and H. Xie, "Nonconforming finite element approximations of the Steklov eigenvalue problem and its lower bound approximations," Applications of Mathematics, vol. 58, no. 2, pp. 129-151, 2013.
[7] Q. Li and Y. Yang, "A two-grid discretization scheme for the Steklov eigenvalue problem," Journal of Applied Mathematics and Computing, vol. 36, no. 1-2, pp. 129-139, 2011.

[8] H. Bi and Y. Yang, "A two-grid method of the non-conforming Crouzeix-Raviart element for the Steklov eigenvalue problem," Applied Mathematics and Computation, vol. 217, no. 23, pp. 9669-9678, 2011.

[9] L. Cao, L. Zhang, W. Allegretto, and Y. Lin, "Multiscale asymptotic method for Steklov eigenvalue equations in composite media," SIAM Journal on Numerical Analysis, vol. 51, no. 1, pp. 273-296, 2013.

[10] H. Xie, "A type of multilevel method for the Steklov eigenvalue problem," IMA Journal of Numerical Analysis, vol. 34, no. 2, pp. 592-608, 2014.

[11] X. Zhang, Y. Yang, and H. Bi, "Spectral method with the tensor-product nodal basis for the Steklov eigenvalue problem," Mathematical Problems in Engineering, vol. 2013, Article ID 650530, 9 pages, 2013.

[12] H. Bi, H. Li, and Y. Yang, "An adaptive algorithm based on the shifted inverse iteration for the Steklov eigenvalue problem," Applied Numerical Mathematics, vol. 105, pp. 64-81, 2016.

[13] I. Babuska and J. Osborn, "Eigenvalue problems in finite element methods (part 1)," in Handbook of Numerical Analysis, P. G. Ciarlet and J. L. Lions, Eds., vol. 2, pp. 640-787, Elsevier Science, North-Holland, The Netherlands, 1991.

[14] I. Babuška and J. E. Osborn, "Finite element-Galerkin approximation of the eigenvalues and eigenvectors of selfadjoint problems," Mathematics of Computation, vol. 52, no. 186, pp. 275-297, 1989.

[15] Y. Yang and H. Bi, "Two-grid finite element discretization schemes based on shifted-inverse power method for elliptic eigenvalue problems," SIAM Journal on Numerical Analysis, vol. 49, no. 4, pp. 1602-1624, 2011.

[16] Y. Yang, H. Bi, J. Han, and Y. Yu, "The shifted-inverse iteration based on the multigrid discretizations for eigenvalue problems," SIAM Journal on Scientific Computing, vol. 37, no. 6, pp. A2583A2606, 2015.

[17] M. Ainsworth and J. T. Oden, A Posteriori Error Estimation in Finite Element Analysis, Wiley-Interscience, New York, NY, USA, 2011.

[18] I. Babuška and W. C. Rheinboldt, "A-posteriori error estimates for the finite element method," International Journal for Numerical Methods in Engineering, vol. 12, no. 10, pp. 1597-1615, 1978. 
[19] P. Morin, R. H. Nochetto, and K. Siebert, "Convergence of adaptive finite element methods," SIAM Review, vol. 44, no. 4, pp. 631-658, 2002.

[20] L. Chen, "iFEM: an innovative finite element methods package in MATLAB," Tech. Rep., University of California, Irvine, Calif, USA, 2009.

[21] Y. J. Li, Y. D. Yang, and H. Bi, “The spectral element method for the Steklov eigenvalue problem," Advanced Materials Research, vol. 853, pp. 631-635, 2014.

[22] E. M. Garau and P. Morin, "Convergence and quasi-optimality of adaptive FEM for Steklov eigenvalue problems," IMA Journal of Numerical Analysis, vol. 31, no. 3, pp. 914-946, 2011. 


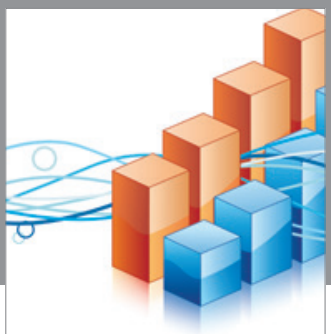

Advances in

Operations Research

vatem alat4

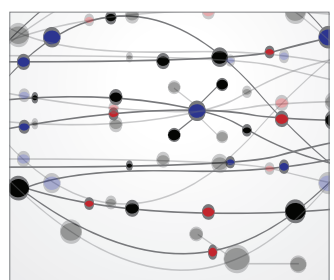

\section{The Scientific} World Journal
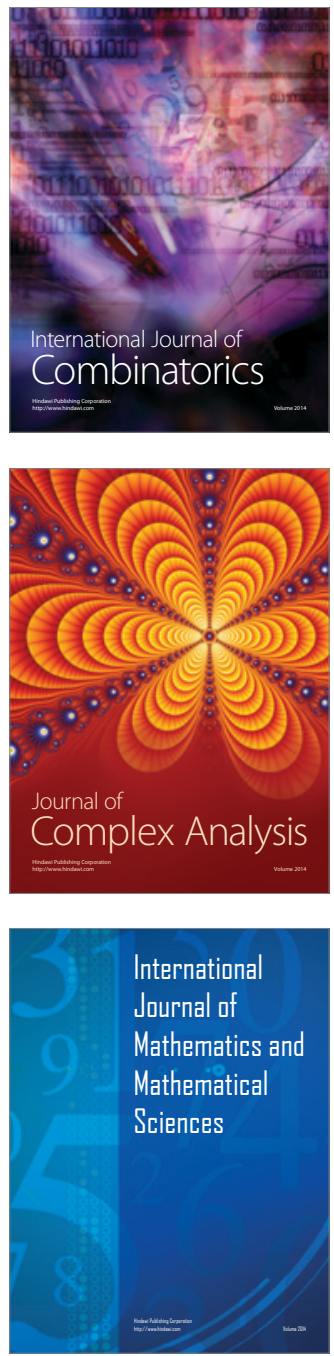
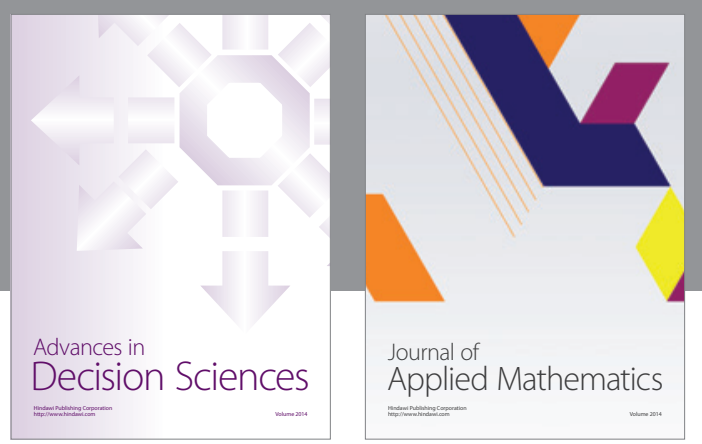

Algebra

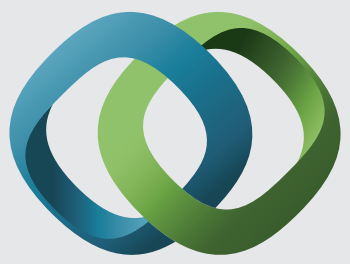

\section{Hindawi}

Submit your manuscripts at

http://www.hindawi.com
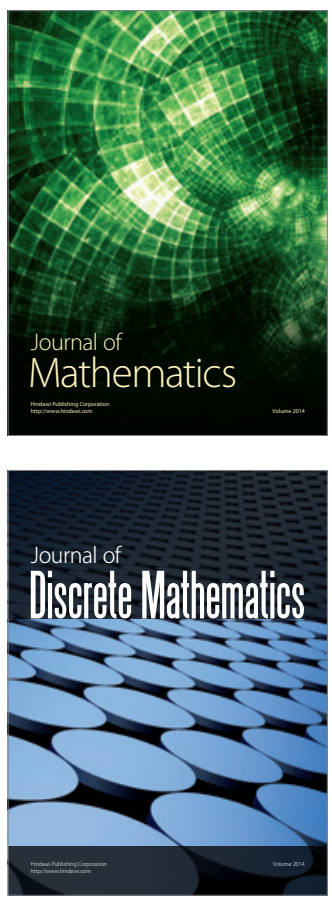

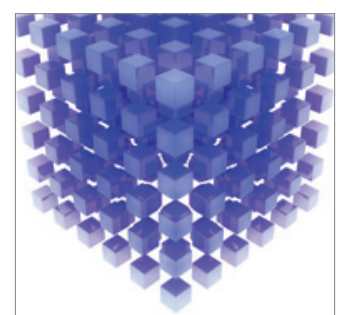

Mathematical Problems in Engineering
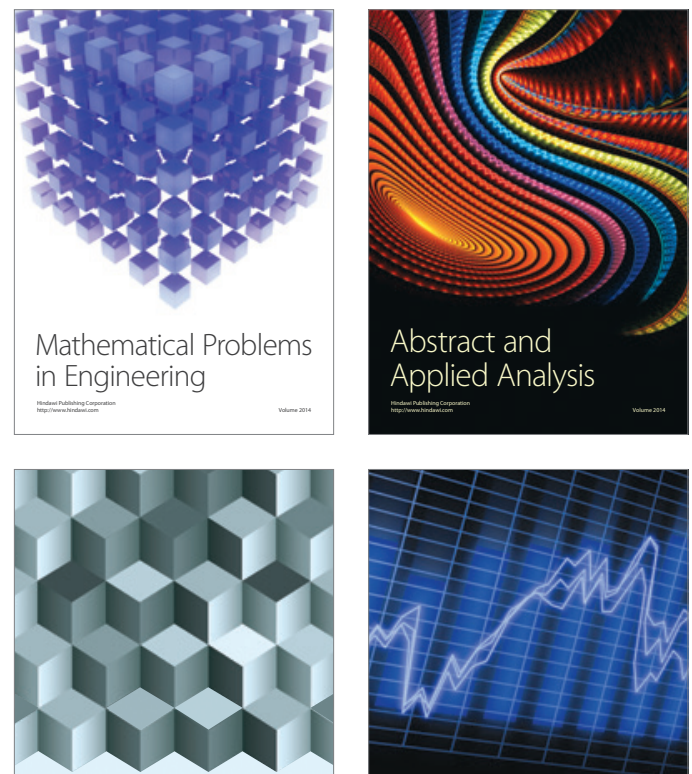

Journal of

Function Spaces

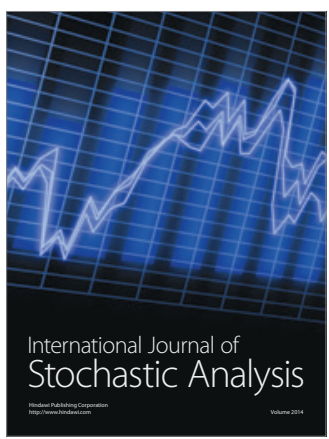

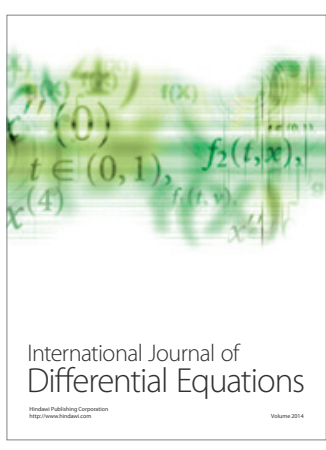
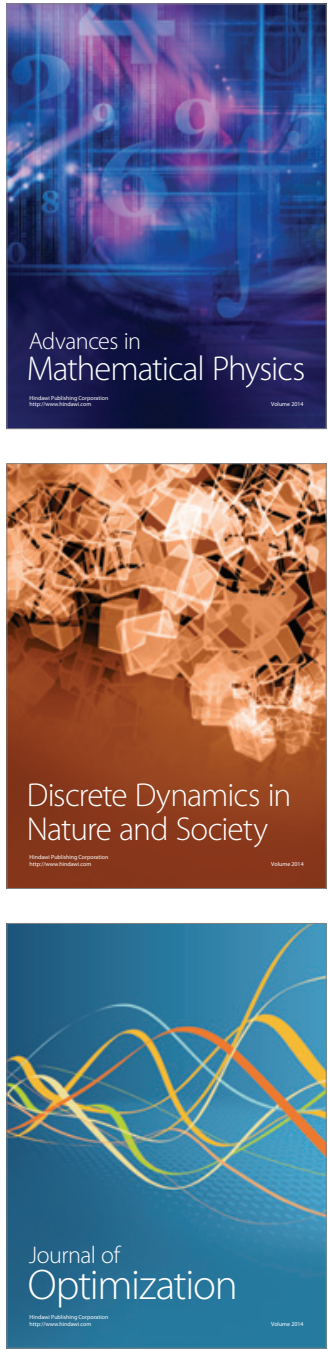